\title{
NGC 6558: A BLUE HORIZONTAL BRANCH MODERATELY METAL-POOR GLOBULAR CLUSTER IN THE BULGE
}

\author{
B. Barbuy, ${ }^{1}$ M. Zoccali, ${ }^{2}$ S. Ortolani, ${ }^{3}$ D. Minniti, ${ }^{2}$ V. Hill, ${ }^{4}$ A. Renzini, ${ }^{5}$ E. Bica, ${ }^{6}$ and A. Gómez ${ }^{4}$ \\ Received 2007 May 6; accepted 2007 July 2
}

\begin{abstract}
We present, for the first time, a detailed abundance analysis of five giant stars in the moderately metal-poor bulge globular cluster NGC 6558. Spectra have been obtained at the VLT with the multifiber spectrograph FLAMES in GIRAFFE mode $(R \sim 22,000)$. The resulting iron abundance is $[\mathrm{Fe} / \mathrm{H}]=-0.97 \pm 0.15$, in good agreement with the metallicity inferred from the slope of the red giant branch, but unusually high for a cluster with such a blue horizontal branch, possibly indicating an old age. A color-magnitude diagram in $V$ and $I$, based on photometry obtained with the Wide-Field Imager at ESO, is also presented. An isochrone of $14 \mathrm{Gyr}$ fits the evolutionary sequences, confirming an old age. NGC 6558 is another "second-parameter" bulge cluster. The metallicity derived is near the end of the low-metallicity tail of the bulge field-star distribution; hence, it presumably formed at the very early stages of the bulge formation. Abundance ratios show enhancements of the $\alpha$-elements oxygen, magnesium, and silicon, with $[\mathrm{O} / \mathrm{Fe}]=+0.38,[\mathrm{Mg} / \mathrm{Fe}]=$ $+0.24,[\mathrm{Si} / \mathrm{Fe}]=+0.23$, and solar calcium and titanium. The $r$-element europium is also enhanced by $[\mathrm{Eu} / \mathrm{Fe}]=$ +0.36 . The odd- $Z$ elements sodium and aluminum, as well as the $s$-elements barium and lanthanum, show solar ratios. A heliocentric radial velocity of $v_{r}^{\text {hel }}=-197.3 \pm 4 \mathrm{~km} \mathrm{~s}^{-1}$ is found for NGC 6558 .
\end{abstract}

Key words: globular clusters: general — globular clusters: individual (NGC 6558)

Online material: color figures

\section{INTRODUCTION}

The formation scenarios of globular clusters (GCs), reviewed by Strader et al. (2005) and Brodie \& Strader (2006), can be summarized as follows: (1) Major merger models suggest the formation of metal-rich GCs during mergers of spiral galaxies that form ellipticals, whereas metal-poor GCs are formed in the original spirals. (2) Multiphase in situ scenarios suppose that metal-poor GCs form in dwarf galaxies within the protogalactic potential, and metalrich GCs form later in a second phase. (3) Accretion scenarios suppose that a massive galaxy core forms metal-rich GCs, and metal-poor ones are accreted from dwarf galaxies. The latter is compatible with an inside-out picture, in which metal-poor clusters would be the first objects to form in the bulge (e.g., Minniti 1995; van den Bergh 1993). Finally, Bekki (2005) compared models of the Galaxy halo in a $\Lambda$ CDM universe and the effects of reionization on the Galactic metal-poor clusters and found that very early formation of metal-poor clusters occurred before reionization and was then truncated at $z \approx 15$. In this case, it is not clear if metal-poor clusters could form in the bulge in a later stage.

The bimodal metallicity distribution of GCs in galaxies (e.g., Larsen et al. 2001; Yoon et al. 2006), first pointed out by Morgan (1959), is also found in the Milky Way, with peaks at

\footnotetext{
${ }^{1}$ Universidade de São Paulo, IAG, Rua do Matão 1226, Cidade Universitária, São Paulo 05508-900, Brazil; barbuy@astro.iag.usp.br.

${ }^{2}$ Departamento de Astronomía y Astrofísica, Pontificia Universidad Católica de Chile, Casilla 306, Santiago 22, Chile; mzoccali@astro.puc.cl, dante@astro .puc.cl.

${ }^{3}$ Dipartimento di Astronomia, Università di Padova, Vicolo dell'Osservatorio 5, I-35122 Padova, Italy; sergio.ortolani@unipd.it.

${ }^{4}$ GEPI, Observatoire de Paris, CNRS, Université Paris Diderot, Place Jules Janssen,92190 Meudon, France; vanessa.hill@obspm.fr, anita.gomez@obspm .fr.

${ }^{5}$ Osservatorio Astronomico di Padova, Vicolo dell'Osservatorio 2, I-35122 Padova, Italy; alvio.renzini@oapd.inaf.it.

${ }^{6}$ Departamento de Astronomia, Universidade Federal do Rio Grande do Sul, CP 15051, Porto Alegre 91501-970, Brazil; bica@if.ufrgs.br.
}

$[\mathrm{Fe} / \mathrm{H}]=-0.5$ and -1.6 (e.g., Côté 1999). Evidence for a third peak at $[\mathrm{Fe} / \mathrm{H}] \approx-1.0$, already seen in Côté (1999), appears to be reinforced in recent years. The 16 clusters located within $5^{\circ} \times 5^{\circ}$ of the Galactic center were reviewed by Barbuy et al. (1998) based on color-magnitude analyses. Four of them have been clearly found to be metal-rich, with $[\mathrm{Fe} / \mathrm{H}] \gtrsim-0.5$ : NGC 6528, NGC 6553, Liller 1, and Terzan 5 (Zoccali et al. 2004; Alves-Brito et al. 2006; Meléndez et al. 2003; Origlia et al. 2002; Origlia \& Rich 2004 ), and two clearly metal-poor, with $[\mathrm{Fe} / \mathrm{H}] \lesssim-1.6$ : Terzan 4 and Terzan 9 (Origlia \& Rich 2004; Ortolani et al. 1999b). Several among the other 10 appear to be moderately metal-poor, namely, HP $1([\mathrm{Fe} / \mathrm{H}]=-1.0$; Barbuy et al. 2006b); Terzan 1, based both on Hubble Space Telescope (HST) color-magnitude diagrams (CMDs) $([\mathrm{Fe} / \mathrm{H}]=-1.3 \text {; Ortolani et al. 1999a })^{7}$ and on lowresolution spectra $([\mathrm{Fe} / \mathrm{H}]=-1.3$; Idiart et al. 2002); UKS 1 and Palomar $6([\mathrm{Fe} / \mathrm{H}]=-0.5$ from the CMDs in Barbuy et al. 1998) were studied with high-resolution spectroscopy by Origlia et al. (2005) and Lee et al. (2004) and found to have $[\mathrm{Fe} / \mathrm{H}]=$ -0.78 and -1.0 , respectively. Finally, NGC 6522 is estimated to have $-1.3<[\mathrm{Fe} / \mathrm{H}]<-1.0$ (Terndrup et al. 1998; Castro et al. 1995). For the clusters Terzan 2, Djorgovski 1, Terzan 6, and ESO 456-SC38, no spectroscopic analyses available have been carried out, and their CMDs suggest $[\mathrm{Fe} / \mathrm{H}] \sim-0.5$ (Barbuy et al. 1998). It is important to note that the moderately metal-poor clusters located in the Galactic bulge would not be detectable in external galaxies due to crowding.

The GC NGC 6558 is located at $\alpha=18^{\mathrm{h}} 10^{\mathrm{m}} 17.6^{\mathrm{s}}, \delta=$ $-31^{\circ} 45^{\prime} 50^{\prime \prime}$ (J2000.0) and projected at about $-6^{\circ}$ from the Galactic center on the minor axis $\left(l=0.201^{\circ}, b=-6.025^{\circ}\right)$. NGC 6558 was studied by Rich et al. (1998) in $V$ and $I$ photometry, where a reddening of $E(B-V)=0.46, E(V-I)=0.6$, and $A_{V}=1.42$, an apparent and absolute distance modulus of $(\mathrm{m}-$ $M)=15.55,(m-M)_{0}=14.13$, and a distance from the Sun of

\footnotetext{
7 The first, overestimated value by Ortolani et al. (1993) was due to high contamination by bulge field stars.
} 

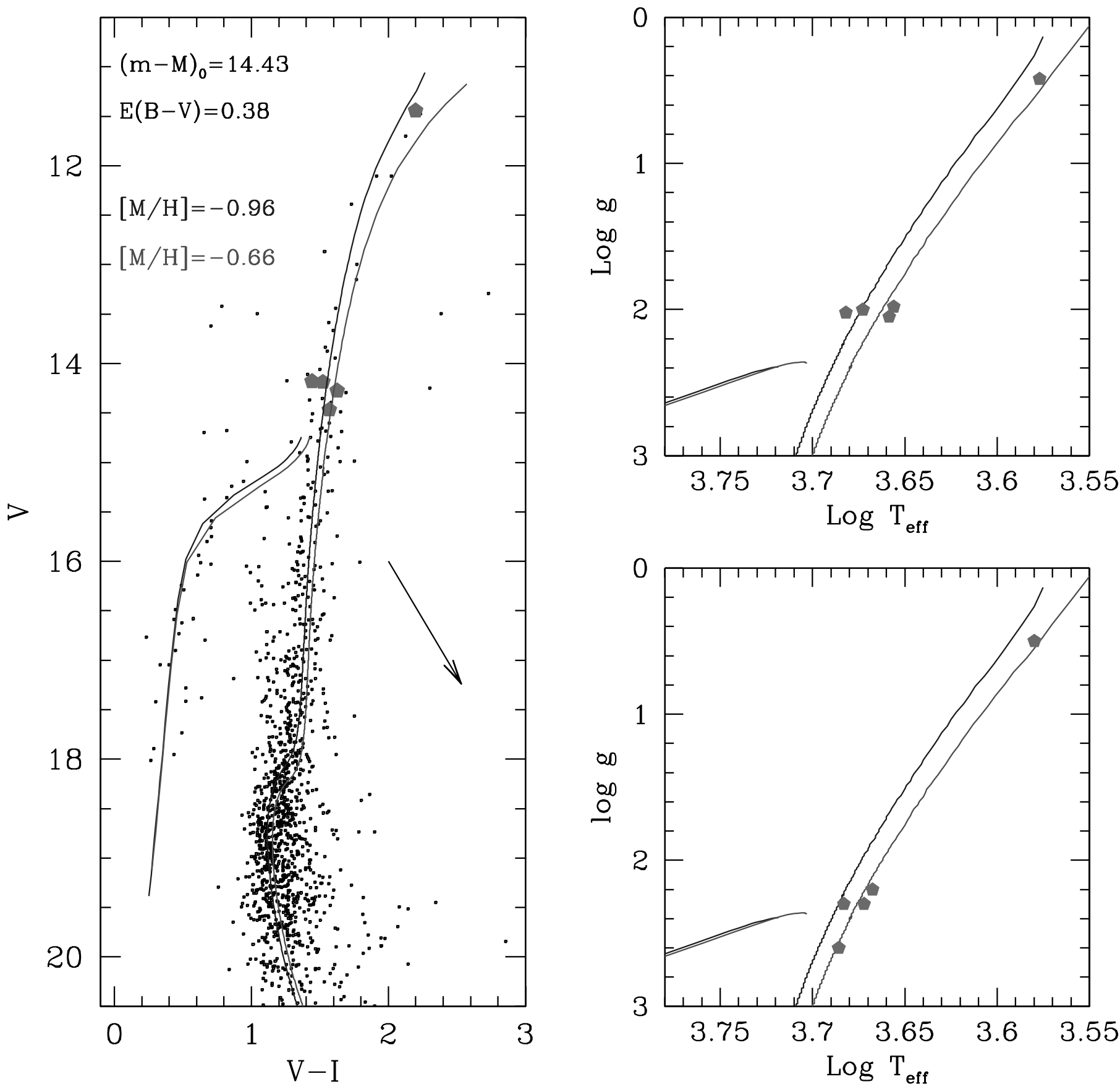

Fig. 1. $-V$ vs. $V-I$ CMD of NGC 6558 with 14 Gyr Teramo isochrones for metallicities $Z=0.002$ and 0.004 overplotted. [See the electronic edition of the Journal for a color version of this figure.]

$d_{\odot}=6.4 \mathrm{kpc}$ and $R_{\mathrm{GC}}=1.8 \mathrm{kpc}$ from the Galactic center were estimated. The cluster is very concentrated, with post-core collapse structure (Trager et al. 1995).

In the present work we analyze high-resolution spectra of five giants in NGC 6558, obtained with the Very Large Telescope (VLT) using FLAMES in GIRAFFE mode. These data are part of a sample of 1000 bulge stars collected in four fields from $-3^{\circ}$ to $-12^{\circ}$ along the bulge minor axis, among which a few cluster stars were included.

The observations are described in $\S 2$, while the stellar parameters and iron abundance are presented in $\S 3$. Results are presented in $\S 4$. Finally, $\S 5$ includes some discussion and our conclusions.

\section{OBSERVATIONS}

\subsection{Imaging}

Images of NGC 6558 in Johnson-Cousins $V$ and $I$, obtained with the Wide Field Imager (WFI) at the $2.2 \mathrm{~m}$ ESO-MPI telescope were downloaded from the ESO archive, within the preFLAMES public ESO Imaging Survey. Standard prereduction (bias and flats) was performed with IRAF, while point-spread function (PSF) fitting photometry was carried out using DAOPHOT II and ALLFRAME (Stetson 1994). The photometric calibrations were defined using standard stars from Landolt (1992). The Galactic coordinates of the cluster are $l=0.201^{\circ}, b=-6.025^{\circ}$, located in the so-called Blanco window (Blanco 1988), and the 




FIG. 2.- I-band ACS image showing the location of the present six spectroscopic targets. The extracted image is $\sim 1.43^{\prime} \times 1.43^{\prime}$. North is up, and east is to the left. [See the electronic edition of the Journal for a color version of this figure.]

WFI image of this region contains mostly bulge stars. In order to build a CMD clearly showing the main cluster features we selected stars within a radius of 400 WFI pixels $\left(95^{\prime \prime}\right)$ around the cluster center. The resulting CMD is shown in Figure 1 (left), together with $14 \mathrm{Gyr} Z=0.002$ and 0.004 Teramo isochrones from Pietrinferni et al. (2004). These models have solar-scaled abundances, and the higher abundance is included, since by considering a combination of the $[\mathrm{Fe} / \mathrm{H}]$ and $\alpha$-element enhancements as reported in $\S 4$ a metallicity $[\mathrm{M} / \mathrm{H}]=-0.75$ is found.

A check of the photometry was carried out by comparing the present data with the New Technology Telescope (NTT) data presented in Rich et al. (1998). NTT photometry is about 0.1-0.15 redder than WFI, within the expected uncertainty of both absolute photometric calibrations. The filters at NTT and at WFI are very different. In particular, the filter system at WFI is quite different from the standard one. The color terms in $V$ and $I$ at NTT are on the order of 0.01-0.02, while at WFI the color term in $V$ is around -0.08 to -0.06 , and in $I$ it is about 0.1 , corresponding to a shift of the equivalent wavelength of the system by more than $200 \AA$. The combined color term for the color index is around 0.16 , corresponding to an order of magnitude larger than at NTT. For normal, unreddened stars the wavelength shift is not relevant because it is compensated for by the color terms, but in highly reddened fields a different reddening law should be applied (Selman \& Melnick 2005), and it should be kept in mind that the calibration of the red stars is based on the linear extrapolation of the calibration. It is not clear, however, if this problem completely justifies the 0.15 color difference, and we do not have enough fields containing reddened stars for a careful check. We made several tests of WFI calibrations and found that an important source of calibration error is the "sky concentration." This makes the calibration dependent on the position of the standards on the chip, which is difficult to correct. In the specific case of NGC 6558 another problem is the crowding effect on the aperture corrections. In this case the lower signal-to-noise ratio $(\mathrm{S} / \mathrm{N})$ of the NTT data, as it appears from the comparison of the two CMDs, seems to indicate a potentially better correction at WFI. In conclusion, NTT calibration would be more reliable because of the better filters, but for the aperture correction there is certainly an issue. A full discussion on the comparisons is beyond the scope of this paper, given that we derived photometric parameters as guidelines for further work on spectroscopic parameters.

\subsection{Spectra}

Spectra for about 200 giants in the Blanco window were obtained with FLAMES in GIRAFFE mode at the VLT, within our large program for a spectroscopic characterization of bulge field stars (Zoccali et al. 2006; Lecureur et al. 2007). The four fields of this survey include a few bulge GCs, and some fibers were positioned close to their centers in order to determine consistent abundances for bulge field and cluster stars. Having measured the radial velocity of the 200 spectra in this field, candidate cluster members were identified by selecting stars with radial velocity $V_{R}<-180 \mathrm{~km} \mathrm{~s}^{-1}$ and coordinates within a radius of $0.05^{\circ}$ from the cluster center. Seven candidates were then identified, six of which turned out to have very similar metallicity (lower than the bulk of bulge stars), and hence appeared to belong to NGC 6558. Star B117 looks anomalous in the CMDs and is probably a nonmember, as discussed below. The positions of the five selected member stars in the CMD are shown in Figure 1, while their identifications on an archive HST ACS image are shown in Figure 2. The star IDs are taken from FLAMES, which has provided spectra for 134 stars in each of the bright (B) and faint (F) half of the stars selected in this field. The cluster members turned out to be star B11 (i.e., the 11th spectrum of the bright sample), B64, and so forth, and such designations were kept. The selected stars, together with the 2MASS designations (Skrutskie et al. 2006), coordinates, and VIJHK magnitudes, are listed in Table 1.

One of the six selected stars, star B117, with $V=15.523$ and $I=14.111$ in WFI and $V=15.59$ and $I=14.05$ in NTT, deserves some further discussion. Star B117 appears well defined, well fitted, and with no blends and no variability when compared to the other stars in the field. However, its position looks anomalous in the CMDs, both from WFI and NTT. It is too blue for its luminosity if interpreted as a giant star of the cluster. However, it should not be a bulge field giant because they are much redder, nor a main-sequence (MS) disk star because they are bluer. Possible explanations are (1) it is an evolved disk star; (2) it is a

TABLE 1

Designation Adopted in the Present Work, 2MASs Identification, Coordinates, and Magnitudes of the Target Stars

\begin{tabular}{|c|c|c|c|c|c|c|c|c|}
\hline Star ID & 2MASS ID & $\alpha(\mathrm{J} 2000.0)$ & $\delta(\mathrm{J} 2000.0)$ & $V$ & $I$ & $J$ & $H$ & K \\
\hline 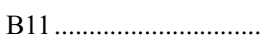 & $18101902-3144506$ & 181019.01 & -314450.64 & 15.902 & 14.275 & 13.136 & 12.440 & 12.280 \\
\hline 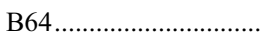 & $18191803-3145435$ & 181018.03 & -314543.55 & 15.623 & 14.180 & 13.064 & 12.456 & 12.277 \\
\hline 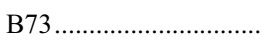 & $18102150-3145268$ & 181021.50 & -314526.77 & 15.709 & 14.187 & 13.128 & 12.449 & 12.316 \\
\hline 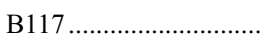 & $18101643-3146005$ & 181016.42 & -314600.55 & 15.523 & 14.111 & 13.148 & 12.569 & 12.491 \\
\hline 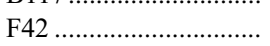 & $18101766-3145393$ & 181017.65 & -314538.93 & 13.642 & 11.442 & 10.054 & 9.049 & 8.812 \\
\hline 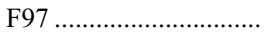 & $18101520-3146014$ & 181015.21 & -314600.67 & 16.037 & 14.467 & 13.183 & 12.481 & 12.338 \\
\hline
\end{tabular}

NoтE.-Units of right ascension are hours, minutes, and seconds, and units of declination are degrees, arcminutes, and arcseconds. 
TABLE 2

LOG OF OBSERVATIONS

\begin{tabular}{|c|c|c|c|c|c|c|c|}
\hline Star & Setup & $\begin{array}{c}\lambda \\
(\mathrm{nm})\end{array}$ & Date & UT & $\mathrm{S} / \mathrm{N}$ & $V_{r}^{\text {obs }}$ & $V_{r}^{\text {hel }}$ \\
\hline \multicolumn{8}{|c|}{ Bright } \\
\hline \multirow[t]{3}{*}{ 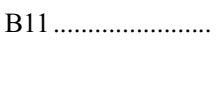 } & HR13 & $612-640$ & 2003 May 31 & $05: 32: 37.740$ & 115 & -207.8 & -198.4 \\
\hline & HR14 & $638-662$ & 2003 May 31 & $07: 20: 32.329$ & 114 & -207.6 & -198.1 \\
\hline & HR15 & $660-686$ & 2003 Jun 1 & $05: 56: 46.114$ & $\ldots$ & -207.7 & -198.3 \\
\hline \multirow[t]{3}{*}{ 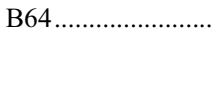 } & HR13 & $612-640$ & 2003 May 31 & $05: 32: 37.740$ & 105 & -204.8 & -195.4 \\
\hline & HR14 & $638-662$ & 2003 May 31 & $07: 20: 32.329$ & 118 & -205.1 & -195.7 \\
\hline & HR15 & $660-686$ & 2003 Jun 1 & $05: 56: 46.114$ & $\ldots$ & -204.3 & -194.9 \\
\hline \multirow[t]{3}{*}{ 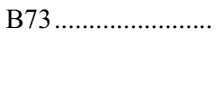 } & HR13 & $612-640$ & 2003 May 31 & $05: 32: 37.740$ & 115 & -205.5 & -196.1 \\
\hline & HR14 & $638-662$ & 2003 May 31 & $07: 20: 32.329$ & 119 & -205.0 & -195.5 \\
\hline & HR15 & $660-686$ & 2003 Jun 1 & $05: 56: 46.114$ & $\ldots$ & -205.8 & -196.4 \\
\hline \multirow[t]{3}{*}{ 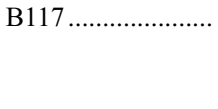 } & HR13 & $612-640$ & 2003 May 31 & $05: 32: 37.740$ & 136 & -210.8 & -201.3 \\
\hline & HR14 & $638-662$ & 2003 May 31 & $07: 20: 32.329$ & 157 & -200.0 & -199.5 \\
\hline & HR15 & $612-640$ & 2003 Jun 1 & $05: 56: 46.114$ & $\ldots$ & -211.0 & -201.3 \\
\hline \multicolumn{8}{|c|}{ Faint } \\
\hline \multirow[t]{3}{*}{ 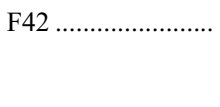 } & HR13 & $612-640$ & 2003 Jun 1 & $07: 12: 15.204$ & 130 & -207.9 & -198.4 \\
\hline & HR14 & $638-662$ & 2003 Jun 27 & 03:54:01.305 & 108 & -207.1 & -197.6 \\
\hline & HR15 & $660-686$ & 2003 Jun 27 & $01: 53: 37.509$ & $\ldots$ & -208.0 & -198.6 \\
\hline \multirow[t]{3}{*}{ F97 ......................... } & HR13 & $612-640$ & 2003 Jun 1 & $07: 12: 15.204$ & 62 & -208.2 & -198.8 \\
\hline & HR14 & $638-662$ & 2003 Jun 27 & 03:54:01.305 & 78 & -207.1 & -197.7 \\
\hline & HR15 & $660-686$ & 2003 Jun 27 & $01: 53: 37.509$ & $\ldots$ & -209.5 & -200.0 \\
\hline
\end{tabular}

peculiar evolved blue straggler; or (3) it is an unresolved blend (or a binary) of a giant and a horizontal branch (HB) star. We have therefore excluded star B117 from the sample because our analysis could turn out to be inappropriate, but a few comments about it are given in the text.

The spectra cover the wavelength range 6100-6860 $\AA$, through the GIRAFFE setups HR13, HR14, and HR15 and at a resolution $R=22,000$. A log of observations is given in Table $2 . \mathrm{S} / \mathrm{N}$ values were measured along the spectra, and a mean from several measurements at different wavelengths in each setup is given in Table 2. No clear continuum window could be identified in the spectra from the HR15 setup; hence, $\mathrm{S} / \mathrm{N}$ values are not measured in this region. The spectra were flat-fielded, optimally extracted, and wavelength calibrated with the GIRAFFE Base-Line Data Reduction Software pipeline. ${ }^{8}$ Spectra extracted from different frames were then summed, and the final spectra of $\sim 15$ fibers positioned on empty sky regions were further combined and subtracted from each star spectrum. The equivalent widths were measured using the automatic code DAOSPEC developed by P. Stetson \& E. Pancino (2007, in preparation).

A radial velocity $v_{r}=-206.8 \pm 1.5 \mathrm{~km} \mathrm{~s}^{-1}$ or heliocentric $v_{r}^{\text {hel }}=-197.3 \pm 4 \mathrm{~km} \mathrm{~s}^{-1}$ was found for NGC 6558, in very

\footnotetext{
${ }^{8}$ Available from http://girbldrs.sourceforge.net.
}

good agreement with the value of $-197.2 \pm 1.6$ reported by Harris (1996).

\section{STELLAR PARAMETERS}

Effective temperatures $T_{\text {eff }}$ were derived from the $V-K$, $V-I$, and $J-K$ colors using the calibrations by Alonso et al. (1999) and adopting $(V-I)_{\mathrm{C}}=0.778(V-I)_{\mathrm{J}}$ from Bessell (1979). The $J H K_{S}$ magnitudes and colors were transformed from the 2MASS system (Skrutskie et al. 2006) ${ }^{10}$ to the CIT (California Institute of Technology) system, and from this to the TCS (Telescopio Carlos Sánchez) system, using the relations established by Carpenter (2001) and Alonso et al. (1998). The $T_{\text {eff }}$ values derived from each color are listed in Table 3.

The above $T_{\text {eff }}$ values were used to derive the gravity $\log g$ from the classical relation

$$
\log g_{*}=4.44+4 \log \frac{T_{*}}{T_{\odot}}+0.4\left(M_{\mathrm{bol}}-4.75\right)+\log \frac{M_{*}}{M_{\odot}},
$$

adopting $T_{\odot}=5770 \mathrm{~K}, M_{*}=0.85 M_{\odot}$, and $M_{\text {bol } \odot}=4.75$. A distance modulus of $(m-M)_{0}=14.43$ and a mean reddening of $E(B-V)=0.38$ were derived from a comparison with the

\footnotetext{
9 Updated at http://www.physics.mcmaster.ca/Globular.html.

${ }^{10}$ See http://www.ipac.caltech.edu/2mass/releases/allsky/.
}

TABLE 3

Dereddened Colors and Derived Photometric Effective Temperatures, Bolometric Corrections, and Gravities

\begin{tabular}{|c|c|c|c|c|c|c|c|c|c|}
\hline Star & $V-I$ & $V-K$ & $J-K$ & $T(V-I)$ & $T(V-K)$ & $T(J-K)$ & $T_{\text {mean }}$ & $\mathrm{BC}_{V}$ & $\log g$ \\
\hline 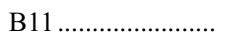 & 1.442 & 2.573 & 0.630 & 4487 & 4515 & 4584 & 4529 & -0.47 & 1.98 \\
\hline  & 1.205 & 2.298 & 0.563 & 4870 & 4757 & 4798 & 4808 & -0.32 & 2.03 \\
\hline 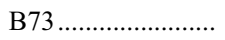 & 1.307 & 2.345 & 0.587 & 4694 & 4709 & 4718 & 4707 & -0.37 & 2.01 \\
\hline 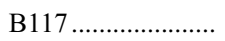 & 1.165 & 1.985 & 0.439 & 4944 & 5103 & 5280 & 5109 & -0.20 & 2.14 \\
\hline 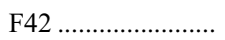 & 2.178 & 3.779 & 1.000 & 3770 & 3823 & 3732 & 3775 & -1.30 & 0.42 \\
\hline F97 ................... & 1.368 & 2.651 & 0.619 & 4596 & 4452 & 4616 & 4555 & -0.45 & 2.05 \\
\hline
\end{tabular}


TABLE 4

Fe i and Fe it Lines Used, Equivalent Widths, and Resulting Fe Abundance Line-by-Line

\begin{tabular}{|c|c|c|c|c|c|c|c|c|c|c|c|c|c|c|}
\hline \multirow[b]{2}{*}{ ION } & \multirow[b]{2}{*}{$\begin{array}{c}\lambda \\
(\AA)\end{array}$} & \multirow[b]{2}{*}{$\begin{array}{c}\chi_{\mathrm{ex}} \\
(\mathrm{eV})\end{array}$} & \multirow[b]{2}{*}{$C_{6}$} & \multirow[b]{2}{*}{$\log g f$} & \multicolumn{2}{|c|}{ B11 } & \multicolumn{2}{|c|}{ B64 } & \multicolumn{2}{|c|}{ B73 } & \multicolumn{2}{|c|}{ F42 } & \multicolumn{2}{|c|}{ F97 } \\
\hline & & & & & $\begin{array}{c}\text { EW } \\
(\mathrm{m} \AA)\end{array}$ & {$[\mathrm{Fe} / \mathrm{H}]$} & $\begin{array}{c}\text { EW } \\
(\mathrm{m} \AA)\end{array}$ & {$[\mathrm{Fe} / \mathrm{H}]$} & $\begin{array}{c}\mathrm{EW} \\
(\mathrm{m} \AA)\end{array}$ & {$[\mathrm{Fe} / \mathrm{H}]$} & $\begin{array}{c}\text { EW } \\
(\mathrm{m} \AA)\end{array}$ & {$[\mathrm{Fe} / \mathrm{H}]$} & $\begin{array}{c}\mathrm{EW} \\
(\mathrm{m} \AA)\end{array}$ & {$[\mathrm{Fe} / \mathrm{H}]$} \\
\hline Fe II .............. & 6149.26 & 3.89 & $0.34 \mathrm{E}-32$ & -2.69 & 27.0 & -0.91 & 28.1 & -0.83 & 34.5 & -0.57 & $\ldots$ & $\ldots$ & 32.7 & -0.84 \\
\hline Fe II .............. & 6247.56 & 3.89 & $0.33 \mathrm{E}-32$ & -2.30 & $\ldots$ & $\ldots$ & $\ldots$ & $\ldots$ & 41.1 & -0.79 & $\ldots$ & $\ldots$ & 42.8 & -0.98 \\
\hline Fe II .................. & 6432.68 & 2.89 & $0.24 \mathrm{E}-32$ & -3.57 & 37.1 & -0.93 & 31.0 & -1.00 & 41.1 & -0.69 & 24.2 & -1.06 & 38.2 & -0.93 \\
\hline Fe II ................. & 6456.39 & 3.90 & $0.32 \mathrm{E}-32$ & -2.05 & 50.7 & -0.96 & 46.0 & -1.00 & 55.1 & -0.69 & 34.2 & -0.89 & $\ldots$ & $\ldots$ \\
\hline Fe II .............. & 6516.08 & 2.89 & $0.25 \mathrm{E}-32$ & -3.450 & 45.1 & -0.88 & 36.2 & -0.99 & 42.9 & -0.77 & 28.6 & -1.03 & $\ldots$ & $\ldots$ \\
\hline Fe I ................... & 6151.62 & 2.18 & $0.81 \mathrm{E}-32$ & -3.299 & 68.9 & -1.12 & 49.4 & -1.14 & 70.8 & -0.91 & 128.9 & -0.85 & 55.1 & -1.14 \\
\hline Fe I ................. & 6159.38 & 4.61 & $0.13 \mathrm{E}-30$ & -1.97 & $\ldots$ & $\ldots$ & 7.1 & -0.80 & 8.1 & -0.82 & $\ldots$ & $\ldots$ & $\ldots$ & $\ldots$ \\
\hline Fe ${ }_{1} \ldots \ldots \ldots \ldots \ldots$ & 6165.36 & 4.14 & $0.77 \mathrm{E}-31$ & -1.549 & 31.0 & -1.13 & 26.4 & -1.06 & 30.1 & -1.08 & 41.8 & -1.28 & $\ldots$ & $\ldots$ \\
\hline Fe I ................... & 6173.34 & 2.22 & $0.84 \mathrm{E}-32$ & -2.879 & 88.2 & -1.13 & 70.8 & -1.07 & 91.6 & -0.85 & 140.8 & -0.98 & 71.1 & -1.21 \\
\hline Fe I .................. & 6180.20 & 2.73 & $0.13 E-31$ & -2.784 & 70.0 & -0.92 & 69.8 & -0.57 & $\ldots$ & $\ldots$ & 108.5 & -0.91 & 46.5 & -1.15 \\
\hline Fe I ................. & 6187.99 & 3.94 & $0.30 \mathrm{E}-30$ & -1.718 & 34.4 & -1.14 & 21.5 & -1.25 & 36.8 & -1.01 & 53.2 & -1.19 & 21.2 & -1.31 \\
\hline Fe I .................. & 6200.31 & 2.61 & $0.15 \mathrm{E}-31$ & -2.437 & 102.6 & -0.79 & $\ldots$ & $\ldots$ & $\ldots$ & $\ldots$ & 124.8 & -1.13 & 78.4 & -1.04 \\
\hline Fe I .................. & 6213.43 & 2.22 & $0.30 \mathrm{E}-31$ & -2.646 & 108.4 & -0.97 & 94.5 & -0.78 & $\ldots$ & $\ldots$ & 141.1 & -1.21 & 92.2 & -1.02 \\
\hline Fe I .................. & 6220.78 & 3.88 & $0.13 E-30$ & -2.46 & $\ldots$ & $\ldots$ & 18.1 & -0.67 & 13.2 & -0.95 & $\ldots$ & $\ldots$ & $\ldots$ & $\ldots$ \\
\hline Fe I ................. & 6226.74 & 3.88 & $0.13 \mathrm{E}-30$ & -2.202 & 19.7 & -1.06 & 17.6 & -0.95 & 23.1 & -0.90 & 27.6 & -1.30 & 16.3 & -1.04 \\
\hline Fe I ................. & 6240.65 & 2.22 & $0.10 \mathrm{E}-31$ & -3.388 & 72.7 & -0.92 & 55.9 & -0.88 & 68.4 & -0.83 & 107.9 & -1.12 & 47.2 & -1.15 \\
\hline Fe ${ }_{1} \ldots \ldots \ldots \ldots \ldots$ & 6246.32 & 3.60 & $0.12 \mathrm{E}-30$ & -0.956 & 104.7 & -1.00 & 98.1 & -0.78 & 104.2 & -0.83 & $\ldots$ & $\ldots$ & 95.5 & -0.97 \\
\hline Fe I ................... & 6252.56 & 2.40 & $0.12 \mathrm{E}-31$ & -1.687 & 139.1 & -1.14 & 124.5 & -0.94 & 132.5 & -1.03 & $\ldots$ & $\ldots$ & 129.9 & -1.02 \\
\hline Fe I ................... & 6254.25 & 2.28 & $0.13 \mathrm{E}-31$ & -2.480 & 124.2 & -0.76 & $\ldots$ & $\ldots$ & 124.9 & -0.56 & 152.0 & -1.13 & 115.1 & -0.69 \\
\hline Fe I ................. & 6270.23 & 2.86 & $0.15 \mathrm{E}-31$ & -2.711 & 56.4 & -1.08 & 50.8 & -0.90 & 60.9 & -0.86 & $\ldots$ & $\ldots$ & 58.9 & -0.84 \\
\hline Fe I .................. & 6271.28 & 3.32 & $0.89 \mathrm{E}-31$ & -2.957 & 16.0 & -1.10 & 11.7 & -1.06 & 23.8 & -0.81 & 40.5 & -1.10 & $\ldots$ & $\ldots$ \\
\hline Fe I ................... & 6297.79 & 2.22 & $0.82 \mathrm{E}-32$ & -2.740 & 88.7 & -1.27 & 72.0 & -1.20 & 86.8 & -1.10 & 141.5 & -1.13 & 80.9 & -1.17 \\
\hline Fe I .................. & 6301.50 & 3.65 & $0.23 \mathrm{E}-31$ & -0.720 & 93.3 & -1.40 & 82.7 & -1.28 & 88.5 & -1.32 & $\ldots$ & $\ldots$ & 83.7 & -1.39 \\
\hline Fe I .................. & 6302.50 & 3.69 & $0.23 \mathrm{E}-31$ & -0.91 & $\ldots$ & $\ldots$ & 70.2 & -1.31 & 76.5 & -1.33 & $\ldots$ & $\ldots$ & $\ldots$ & $\ldots$ \\
\hline 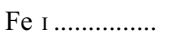 & 6311.50 & 2.83 & $0.14 \mathrm{E}-31$ & -3.224 & 28.9 & -1.11 & 25.8 & -0.95 & 37.1 & -0.85 & 79.6 & -0.88 & 41.5 & -0.69 \\
\hline Fe I ................... & 6315.31 & 4.14 & $0.30 \mathrm{E}-31$ & -1.230 & 57.9 & -0.95 & 44.4 & -1.00 & 48.0 & -1.04 & 79.4 & -0.90 & 62.6 & -0.72 \\
\hline Fe I .................. & 6315.81 & 4.08 & $0.66 \mathrm{E}-31$ & -1.712 & 28.0 & -1.11 & 22.5 & -1.07 & 32.6 & -0.94 & 46.0 & -1.13 & 32.0 & -0.90 \\
\hline Fe I ................... & 6322.69 & 2.59 & $0.14 \mathrm{E}-31$ & -2.426 & 101.1 & -0.88 & 82.1 & -0.84 & 91.9 & -0.85 & 135.7 & -0.98 & 87.3 & -0.90 \\
\hline Fe I ................. & 6335.33 & 2.20 & $0.80 \mathrm{E}-32$ & -2.229 & 125.3 & -1.11 & 109.4 & -0.93 & 121.2 & -0.95 & 186.7 & -0.90 & $\ldots$ & $\ldots$ \\
\hline Fe I .................. & 6336.82 & 3.69 & $0.13 E-30$ & -1.053 & 92.2 & -1.04 & 87.4 & -0.80 & 101.2 & -0.69 & $\ldots$ & $\ldots$ & 95.8 & -0.77 \\
\hline Fe I ................... & 6344.15 & 2.43 & $0.12 \mathrm{E}-31$ & -2.922 & 82.4 & -0.95 & 70.4 & -0.80 & 79.1 & -0.82 & 141.8 & -0.63 & $\ldots$ & $\ldots$ \\
\hline Fe I .................. & 6355.03 & 2.84 & $0.30 \mathrm{E}-31$ & -2.40 & $\ldots$ & $\ldots$ & $\ldots$ & $\ldots$ & 94.4 & -0.51 & 134.9 & -0.64 & 85.1 & -0.67 \\
\hline Fe I .................. & 6392.54 & 2.28 & $0.11 \mathrm{E}-31$ & -4.03 & $\ldots$ & $\ldots$ & 20.4 & -0.94 & 37.5 & -0.72 & 79.5 & -0.93 & 32.7 & -0.72 \\
\hline Fe I ................... & 6392.54 & 2.28 & $0.3 \mathrm{E}-31$ & -4.03 & $\ldots$ & $\ldots$ & 19.6 & -0.96 & 24.1 & -1.00 & 63.1 & -1.20 & 17.5 & -1.08 \\
\hline Fe I .................. & 6393.60 & 2.43 & $0.12 \mathrm{E}-31$ & -1.615 & 159.0 & -0.88 & 134.1 & -0.84 & 157.1 & -0.73 & 195.5 & -1.05 & 115.3 & -1.35 \\
\hline Fe I ................... & 6393.60 & 2.43 & $0.3 \mathrm{E}-31$ & -1.615 & 122.2 & -1.50 & 124.2 & -1.01 & 140.4 & -0.97 & $\ldots$ & $\ldots$ & 124.3 & -1.18 \\
\hline Fe I ................... & 6419.95 & 4.73 & $0.15 \mathrm{E}-30$ & -0.250 & 58.3 & -1.21 & 58.5 & -1.02 & 60.7 & -1.07 & 74.4 & -1.11 & 49.5 & -1.26 \\
\hline Fe I ................. & 6430.85 & 2.18 & $0.77 \mathrm{E}-32$ & -2.005 & 129.5 & -1.30 & 115.2 & -1.08 & 129.8 & -1.05 & $\ldots$ & $\ldots$ & 133.1 & -0.94 \\
\hline Fe I ................... & 6475.62 & 2.56 & $0.15 \mathrm{E}-31$ & -2.94 & $\ldots$ & $\ldots$ & 51.4 & -1.02 & 65.3 & -0.93 & 111.0 & -1.02 & $\ldots$ & $\ldots$ \\
\hline Fe I ........ & 6481.87 & 2.28 & $0.11 \mathrm{E}-31$ & -2.984 & 83.2 & -1.08 & 60.7 & -1.13 & $\ldots$ & $\ldots$ & 128.7 & -1.08 & 133.1 & -0.94 \\
\hline Fe I ................... & 6498.94 & 0.96 & $0.49 \mathrm{E}-32$ & -4.699 & 85.3 & -1.04 & 63.5 & -0.98 & $\ldots$ & $\ldots$ & 173.6 & -0.64 & 77.8 & -0.86 \\
\hline Fe I ................. & 6518.37 & 2.83 & $0.13 \mathrm{E}-31$ & -2.748 & 64.8 & -0.95 & 46.8 & -0.99 & 63.6 & -0.82 & 102.1 & -0.96 & 59.6 & -0.84 \\
\hline Fe I .................. & 6533.93 & 4.56 & $0.16 \mathrm{E}-30$ & -1.453 & 23.8 & -0.90 & 22.5 & -0.79 & 26.9 & -0.76 & 34.2 & -0.94 & $\ldots$ & $\ldots$ \\
\hline Fe I .................. & 6569.22 & 4.73 & $0.13 E-30$ & -0.422 & 53.2 & -1.14 & 45.1 & -1.12 & 58.8 & -0.94 & 79.0 & -0.86 & $\ldots$ & $\ldots$ \\
\hline Fe I .................. & 6574.23 & 0.99 & $0.56 \mathrm{E}-32$ & -5.042 & 58.1 & -1.12 & 47.6 & -0.91 & 57.0 & -0.97 & 143.6 & -0.82 & 44.0 & -1.08 \\
\hline Fe I .................. & 6575.02 & 2.59 & $0.14 \mathrm{E}-31$ & -2.824 & 74.3 & -1.01 & 54.0 & -1.06 & 73.1 & -0.86 & 130.7 & -0.73 & $\ldots$ & $\ldots$ \\
\hline Fe I ................. & 6591.31 & 4.59 & $0.12 \mathrm{E}-30$ & -2.06 & $\ldots$ & $\ldots$ & 11.3 & -0.52 & $\ldots$ & $\ldots$ & $\ldots$ & $\ldots$ & $\ldots$ & $\ldots$ \\
\hline Fe I .................. & 6593.87 & 2.43 & $0.12 \mathrm{E}-31$ & -2.422 & 106.3 & -1.02 & $\ldots$ & $\ldots$ & 109.3 & -0.73 & $\ldots$ & $\ldots$ & 94.0 & -1.00 \\
\hline Fe I .................. & 6597.56 & 4.80 & $0.15 \mathrm{E}-30$ & -1.061 & 21.2 & -1.07 & 21.7 & -0.93 & 27.1 & -0.87 & 35.2 & -0.97 & $\ldots$ & $\ldots$ \\
\hline Fe I ................... & 6608.03 & 2.28 & $0.10 \mathrm{E}-31$ & -4.038 & 31.6 & -0.94 & 16.0 & -1.07 & 25.1 & -0.98 & 82.7 & -0.88 & 21.9 & -0.96 \\
\hline Fe I ................. & 6678.00 & 2.69 & $0.30 \mathrm{E}-31$ & -1.420 & 134.0 & -1.20 & 127.3 & -0.89 & $\ldots$ & $\ldots$ & $\ldots$ & $\ldots$ & $\ldots$ & $\ldots$ \\
\hline Fe I .................. & 6703.57 & 2.76 & $0.12 \mathrm{E}-31$ & -3.15 & $\ldots$ & $\ldots$ & 40.4 & -0.81 & $\ldots$ & $\ldots$ & 83.7 & -1.02 & 37.8 & -0.94 \\
\hline Fe I .................. & 6705.11 & 4.61 & $0.30 \mathrm{E}-31$ & -1.060 & 30.8 & -1.08 & 22.5 & -1.13 & 41.9 & -0.78 & 35.2 & -1.24 & 43.4 & -0.67 \\
\hline Fe I ................... & 6710.32 & 1.48 & $0.64 \mathrm{E}-32$ & -4.874 & 34.9 & -1.06 & 26.6 & -0.92 & 30.5 & -1.02 & 101.7 & -0.98 & $\ldots$ & $\ldots$ \\
\hline Fe I ................. & 6713.75 & 4.80 & $0.16 \mathrm{E}-30$ & -1.602 & 6.6 & -1.12 & 10.0 & -0.81 & $\ldots$ & $\ldots$ & 15.7 & -0.93 & $\ldots$ & $\ldots$ \\
\hline Fe I .................. & 6715.38 & 4.59 & $0.11 \mathrm{E}-30$ & -1.638 & 14.6 & -0.95 & 8.3 & -1.10 & 15.8 & -0.87 & 30.0 & -0.81 & $\ldots$ & $\ldots$ \\
\hline Fe I ................... & 6716.24 & 4.56 & $0.79 \mathrm{E}-32$ & -1.927 & 11.3 & -0.82 & $\ldots$ & $\ldots$ & 18.8 & -0.50 & 24.1 & -0.70 & $\ldots$ & $\ldots$ \\
\hline Fe I .................. & 6725.36 & 4.10 & $0.15 \mathrm{E}-30$ & -2.300 & 12.0 & -0.97 & 5.5 & -1.20 & $\ldots$ & $\ldots$ & 23.3 & -1.01 & $\ldots$ & $\ldots$ \\
\hline Fe I .................. & 6726.67 & 4.59 & $0.30 \mathrm{E}-31$ & -1.090 & 22.4 & -1.26 & 14.0 & -1.39 & 37.4 & -0.86 & 36.3 & -1.22 & 32.6 & -0.93 \\
\hline Fe I .................. & 6733.15 & 4.64 & $0.11 \mathrm{E}-30$ & -1.576 & 17.5 & -0.86 & 21.2 & -0.61 & $\ldots$ & $\ldots$ & 16.6 & -1.15 & 22.3 & -0.63 \\
\hline Fe I .................. & 6739.52 & 1.56 & $0.67 \mathrm{E}-32$ & -4.941 & 35.2 & -0.88 & $\ldots$ & $\ldots$ & 31.9 & -0.83 & 78.5 & -1.18 & 33.1 & -0.69 \\
\hline Fe I ................... & 6752.71 & 4.64 & $0.11 \mathrm{E}-30$ & -1.366 & 15.2 & -1.14 & 25.0 & -0.72 & 26.8 & -0.76 & $\ldots$ & $\ldots$ & $\ldots$ & $\ldots$ \\
\hline
\end{tabular}


TABLE 4-Continued

\begin{tabular}{|c|c|c|c|c|c|c|c|c|c|c|c|c|c|c|}
\hline \multirow[b]{2}{*}{ IoN } & \multirow[b]{2}{*}{$\begin{array}{c}\lambda \\
(\AA)\end{array}$} & \multirow[b]{2}{*}{$\begin{array}{c}\chi_{\mathrm{ex}} \\
(\mathrm{eV})\end{array}$} & \multirow[b]{2}{*}{$C_{6}$} & \multirow[b]{2}{*}{$\log g f$} & \multicolumn{2}{|c|}{ B11 } & \multicolumn{2}{|c|}{ B64 } & \multicolumn{2}{|c|}{ B73 } & \multicolumn{2}{|c|}{$\mathrm{F} 42$} & \multicolumn{2}{|c|}{ F97 } \\
\hline & & & & & $\begin{array}{c}\text { EW } \\
(\mathrm{m} \AA)\end{array}$ & {$[\mathrm{Fe} / \mathrm{H}]$} & $\begin{array}{c}\mathrm{EW} \\
(\mathrm{m} \AA)\end{array}$ & {$[\mathrm{Fe} / \mathrm{H}]$} & $\begin{array}{c}\mathrm{EW} \\
(\mathrm{m} \AA)\end{array}$ & {$[\mathrm{Fe} / \mathrm{H}]$} & $\begin{array}{c}\text { EW } \\
(\mathrm{mA})\end{array}$ & {$[\mathrm{Fe} / \mathrm{H}]$} & $\begin{array}{c}\text { EW } \\
(\mathrm{m} \AA)\end{array}$ & {$[\mathrm{Fe} / \mathrm{H}]$} \\
\hline Fe ${ }_{1} \ldots \ldots \ldots \ldots \ldots \ldots$ & 6810.26 & 4.60 & $0.14 \mathrm{E}-30$ & -1.108 & 35.9 & -0.94 & 35.7 & -0.79 & $\ldots$ & $\ldots$ & 58.1 & -0.77 & 31.4 & -0.93 \\
\hline 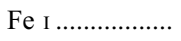 & 6820.37 & 4.64 & $0.16 \mathrm{E}-30$ & -1.311 & 30.3 & -0.80 & 18.4 & -0.97 & $\ldots$ & $\ldots$ & 35.4 & -0.95 & $\ldots$ & $\ldots$ \\
\hline  & 6837.02 & 4.59 & $0.78 \mathrm{E}-32$ & -1.800 & 10.5 & -0.95 & 25.8 & -0.34 & $\ldots$ & $\ldots$ & 25.0 & -0.77 & $\ldots$ & $\ldots$ \\
\hline 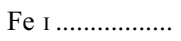 & 6839.83 & 2.56 & $0.13 \mathrm{E}-31$ & -3.451 & 32.4 & -1.18 & 22.2 & -1.16 & 30.5 & -1.12 & 77.1 & -1.15 & 32.5 & -0.99 \\
\hline Fe I .................. & 6841.34 & 4.61 & $0.10 \mathrm{E}-30$ & -0.752 & 49.8 & -1.02 & 44.4 & -0.95 & 45.5 & -1.02 & 53.3 & -1.20 & 35.6 & -1.19 \\
\hline Fe I ............... & 6842.69 & 4.64 & $0.15 \mathrm{E}-30$ & -1.315 & 28.8 & -0.83 & 35.7 & -0.53 & $\ldots$ & $\ldots$ & 42.4 & -0.80 & 16.4 & -1.06 \\
\hline Fe I .................... & 6843.66 & 4.55 & $0.94 \mathrm{E}-31$ & -0.928 & 46.3 & -0.98 & 44.7 & -0.84 & $\ldots$ & $\ldots$ & $\ldots$ & $\ldots$ & $\ldots$ & $\ldots$ \\
\hline Fe I .................. & 6851.63 & 1.60 & $0.73 E-32$ & -5.307 & 12.9 & -1.02 & $\ldots$ & $\ldots$ & $\ldots$ & $\ldots$ & $\ldots$ & $\ldots$ & $\ldots$ & $\ldots$ \\
\hline 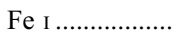 & 6855.71 & 4.39 & $0.10 \mathrm{E}-30$ & -1.819 & 23.5 & -0.75 & 22.0 & -0.64 & $\ldots$ & $\ldots$ & 24.3 & -1.05 & 18.4 & -0.79 \\
\hline Fe I ............... & 6857.25 & 4.08 & $0.53 \mathrm{E}-31$ & -2.156 & 12.9 & -1.11 & 27.8 & -0.52 & $\ldots$ & $\ldots$ & 22.2 & -1.21 & 9.0 & -1.18 \\
\hline Fe I .................. & 6858.15 & 4.61 & $0.10 \mathrm{E}-30$ & -1.055 & 38.4 & -0.93 & 31.7 & -0.92 & 47.4 & -0.68 & 46.0 & -1.04 & 22.7 & -1.18 \\
\hline
\end{tabular}

isochrone in Figure 1. In this same Figure 1 the smaller right panels show the locations of the sample stars in $\log g$ versus $\log T_{\text {eff }}$, for metallicities $Z=0.002$ (top) and 0.004 (bottom) $([\mathrm{M} / \mathrm{H}]=-0.96$ and -0.66).

The $\mathrm{Fe}$ I line list and respective oscillator strengths given by NIST) ${ }^{11}$ were used to derive spectroscopic parameters; if not available, they were obtained from a fit to the solar spectrum as described in Barbuy et al. (1999). Five measurable Fe II lines and their respective oscillator strengths from Biémont et al. (1991), renormalized by J. Meléndez \& B. Barbuy (2007, in preparation), were used to check whether ionization equilibrium was verified. The damping constants were computed where possible, and in particular for most of the $\mathrm{Fe}_{\mathrm{I}}$ lines, using the collisional broadening theory of Barklem et al. (1998, 2000, and references therein). The list of $\mathrm{Fe}$ I and $\mathrm{Fe}$ II lines, together with the $\log g f$ used, equivalent widths, and line-by-line abundances are given in Table 4 . We used only lines with equivalent widths $\mathrm{EW}<200 \mathrm{~m} \AA$.

${ }^{11}$ Available from http://physics.nist.gov/PhysRefData/ASD/.
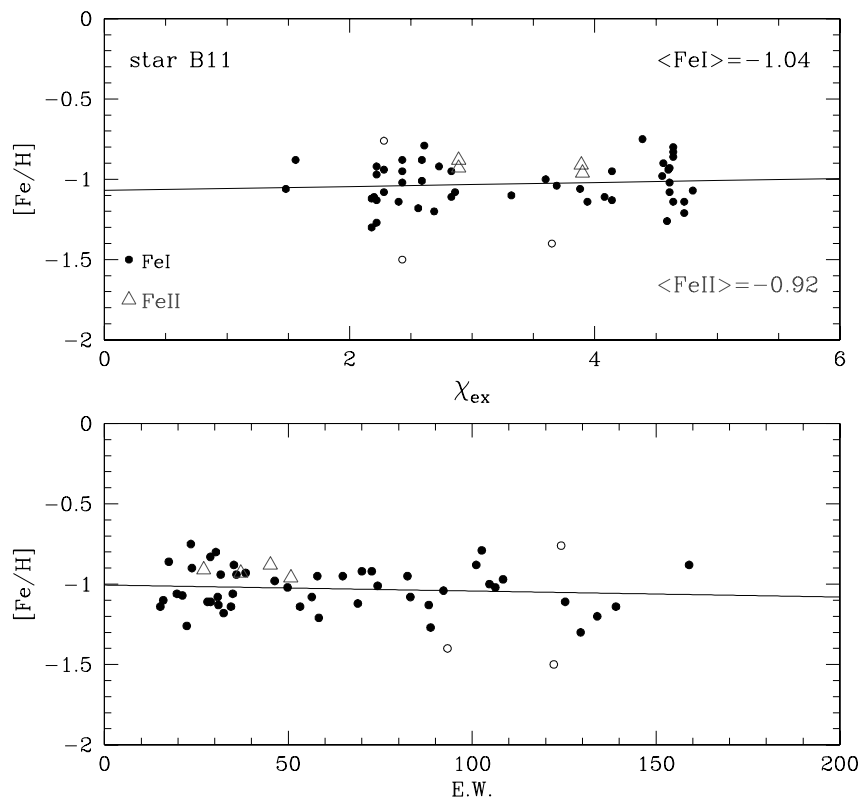

Model atmospheres were interpolated in the newly built OSMARCS grid of spherical models by Gustafsson et al. (2003). ${ }^{12}$ Excitation and ionization equilibria were imposed through curves of growth and the plots of $[\mathrm{Fe} / / \mathrm{H}]$ and $[\mathrm{Fe}$ II $/ \mathrm{H}]$ versus $\chi_{\text {exc }}$ and $W_{\lambda}$, as shown in Figure 3. Microturbulent velocities $v_{t}$ are obtained by imposing the condition that lines of different strengths give the same Fe abundance, as illustrated in Figure 3 (bottom panels). The final adopted stellar parameters and iron abundances are given in Table 5.

Line-by-line spectrum synthesis was carried out to derive elemental abundances, using the code described in Barbuy et al. (2003). The forbidden oxygen line [O I] $\lambda 6300$ could not be used because at the radial velocity of NGC 6558 it falls right on top of a strong telluric line. Since our final spectrum is the combination of several individual exposures at different air masses, we judged it unreliable to try to subtract the telluric line. ${ }^{13}$ We measured instead the oxygen abundance from the other line of the doublet,

\footnotetext{
12 Available from http://www.marcs.astro.uu.se.

13 For these reasons, the preliminary results on the oxygen abundance in NGC 6558 reported in Barbuy et al. (2006a) are superseded by the present results.
}
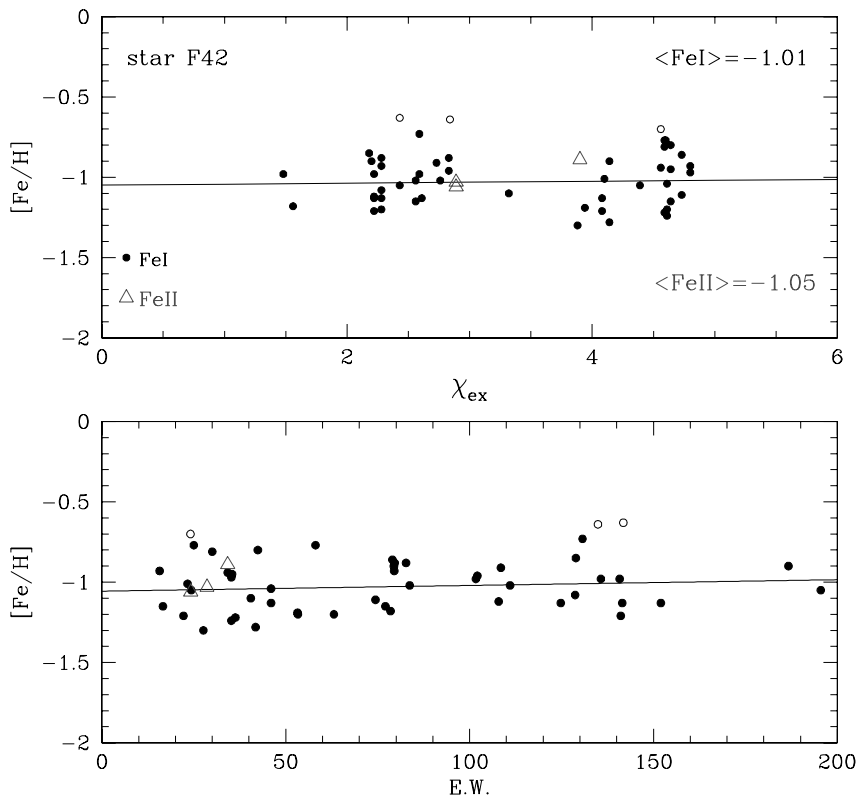

FIG. 3.-Fe I and Fe II abundances vs. $\chi_{\text {exc }}$ and $W_{\lambda}$ for stars B11 and F42. Filled circles, Fe I lines; triangles, Fe II lines; open circles, Fe I lines rejected by sigma clipping. [See the electronic edition of the Journal for a color version of this figure.] 
TABLE 5

Spectroscopic Parameters

\begin{tabular}{|c|c|c|c|c|c|c|}
\hline Star & $T_{\text {eff }}$ & $\log g$ & {$\left[\mathrm{Fe} \mathrm{I}_{\mathrm{I}} / \mathrm{H}\right]$} & {$[\mathrm{Fe}$ II $/ \mathrm{H}]$} & $v_{t}$ & $\begin{array}{c}v_{r}^{\text {hel }} \\
\left(\mathrm{km} \mathrm{s}^{-1}\right)\end{array}$ \\
\hline $\mathrm{B} 11 \ldots$ & 4650 & 2.2 & -1.04 & -0.92 & 1.5 & -198.2 \\
\hline В64...................... & 4850 & 2.6 & -0.94 & -1.00 & 1.2 & -195.3 \\
\hline B73 & 4700 & 2.3 & -0.92 & -0.83 & 1.3 & -196.0 \\
\hline B117 ................... & 5000 & 1.3 & -1.14 & -1.06 & 1.3 & -198.2 \\
\hline F42 .................... & 3800 & 0.5 & -1.01 & -1.05 & 1.65 & -198.2 \\
\hline 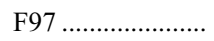 & 4820 & 2.3 & -0.97 & -0.94 & 1.3 & -198.8 \\
\hline
\end{tabular}

[O I] 26363.79 . This line is much weaker, and the results are therefore more uncertain than those derived in other stars from the $\left[\mathrm{O}_{\mathrm{I}}\right] \lambda 6300$ line.

For the odd- $Z$ elements $\mathrm{Al}$ and $\mathrm{Na}$, and the $\alpha$-elements $\mathrm{Mg}, \mathrm{Si}$, $\mathrm{Ca}$, and $\mathrm{Ti}$, the lines and respective atomic constants $\log g f$ and $C_{6}$ are adopted basically from Barbuy et al. (2006b) and Zoccali et al. (2004). However, all lines have been reverified in the solar and Arcturus spectra, and for a few lines a modified $\log g f$ is used as indicated in Table 6. Ionization equilibrium between Ti I and $\mathrm{Ti}$ II is difficult to reach due to uncertainties in the $\log g f$ values of the $\mathrm{Ti}$ II lines reported in Table 7. Ionization equilibrium can be reached if we use the revised Ti II $\log g f$ values from the Vienna Atomic Line Database (VALD; Kupka et al. 1999). These $\log g f$ values do not fit the solar and Arcturus spectra; hence, we preferred the modified values given in Tables 6 and 7 (col. [8]) that fit the solar and Arcturus spectra (as illustrated in Figs. 6 and 7).

It is also important to note that for several $\mathrm{Ca}$ I lines, the revised $\log g f$ values are lower than previously adopted in Barbuy et al. (2006b) and Zoccali et al. (2004): $\Delta \log g f=0.2,0.4,0.2$, $0.16,0.18,0.15,0.25,0.06$, and 0.23 dex were applied to the $\mathrm{Ca}$ I lines at $6156.030,6161.295,6166.440,6169.044,6169.564$, $6464.679,6493.788$, and $6499.654 \AA$, and the result is that $\mathrm{Ca}$ abundances are higher than they would be with the previous $\log g f$ values, by about 0.1 dex in the mean.

The autoionization effect on the wings of the $\mathrm{Ca}$ I $\lambda 6318.61$ and $\lambda 6361.94$ lines has been taken into account in the calculation of the $\mathrm{Mg}$ I triplet at $6318.72,6319.24$, and $6319.49 \AA$, and the [O I] $\lambda 6363.79$ line, by adopting a radiative broadening 10,000 larger than the standard value for the $\mathrm{Ca}$ I line (see also Lecureur et al. 2007).

For lines of the heavy elements Ba II, La II, and Eu II, a hyperfine structure was taken into account, based on the hyperfine constants by Lawler et al. (2001b) for Eu II, Lawler et al. (2001a) for La II, and Biehl (1976) for Ba II. Molecular lines of CN $\left(A^{2} \Pi-X^{2} \Sigma\right), \mathrm{C}_{2}$ Swan $\left(A^{3} \Pi-X^{3} \Pi\right), \mathrm{TiO}\left(A^{3} \Phi-X^{3} \Delta\right) \gamma$, and $\mathrm{TiO}\left(B^{3} \Pi-X^{3} \Delta\right) \gamma^{\prime \prime}$ systems were taken into account. Solar abundances were adopted from Grevesse \& Sauval (1998), except for the value for oxygen, where $\epsilon(\mathrm{O})=8.77$ was assumed, as recommended by Allende Prieto et al. (2001) for the use of onedimensional model atmospheres.

\section{RESULTS}

As shown in Table 5 , we derived a mean metallicity of $[\mathrm{Fe} / \mathrm{H}]=$ $-0.97 \pm 0.15$ for NGC 6558 . Together with HP 1 , they are the highest metallicity clusters with a blue horizontal branch (BHB) but no red horizontal branch (RHB). If interpreted as an age effect (Lee et al. 1994), this would imply that NGC 6558 is one of the oldest clusters in the bulge. This is consistent with the isochrone of 14 Gyr (Fig. 1).

NGC 6558 appears to be similar to other intermediate-metallicity clusters located in the bulge such as, in particular, HP 1 (Ortolani et al. 1997; Barbuy et al. 2006b). Table 8 lists the known clusters with confirmed or probable metallicities around $[\mathrm{Fe} / \mathrm{H}]=$ -1.0 , combined with a clear BHB. NGC 6540 (Bica et al. 1994) could be another candidate if the moderate metallicity is confirmed.

These are all intermediate-metallicity clusters with a BHB, as well as other well-known second-parameter clusters, in particular the well-studied NGC 2808, NGC 6388, and NGC 6441. These latter three clusters show extended HB blue tails, but they also show a well-populated RHB (Rich et al. 1997; Sosin et al. 1997; Bedin et al. 2000). On the contrary, the clusters listed in Table 8 have only a BHB, and in particular HP 1 and NGC 6558, for which highresolution abundance analyses show $[\mathrm{Fe} / \mathrm{H}] \approx-1.0$, could represent a more extreme second-parameter effect, possibly indicating an older age (e.g., Lee et al. 1994).

Line-by-line abundance ratios are given in Table 6 , whereas the final cluster abundances are reported in Table 9. Figures 4 and 5 show the fits to typical $\mathrm{Ca}$ I lines, and Figures 6 and 7 show the fits to $\mathrm{Ti}$ I and $\mathrm{Ti}$ II lines, respectively.

The mean oxygen-to-iron ratio, including all the stars, is $[\mathrm{O} / \mathrm{Fe}]=+0.38$. Rejecting $\mathrm{F} 97$, which shows anomalously high oxygen abundance, the mean would be $[\mathrm{O} / \mathrm{Fe}]=+0.26$, still indicating a clear enhancement of this representative $\alpha$-element. This overabundance is compatible with field stars in the Galaxy (see, e.g., Fig. 5 from Meléndez \& Barbuy [2002], where data on oxygen-to-iron ratios from the $[\mathrm{O} \mathrm{I}] \lambda 6300$ line from the literature were gathered).

The other $\alpha$-elements $\mathrm{Mg}$ and $\mathrm{Si}$ are also enhanced by similar amounts of $[\mathrm{Mg} / \mathrm{Fe}]=+0.24$ and $[\mathrm{Si} / \mathrm{Fe}]=+0.23$. On the other hand, $\mathrm{Ca}$ and Ti show essentially solar ratios, with $[\mathrm{Ca} / \mathrm{Fe}]=+0.05$ and $[\mathrm{Ti} / \mathrm{Fe}]=+0.06$.

The odd- $Z$ elements show solar ratios, with $\mathrm{Na}$ tending to be slightly underabundant. The $r$-element Eu shows enhancement of $[\mathrm{Eu} / \mathrm{Fe}]=+0.36$. The $s$-elements $\mathrm{Ba}$ and La show solar ratios.

\subsection{Star B117}

Spectroscopic parameters for B117 established by imposing excitation and ionization equilibria give $T_{\text {eff }}=5000 \mathrm{~K}, \log g=$ $1.3,[\mathrm{Fe} \mathrm{I} / \mathrm{H}]=-1.14,[\mathrm{Fe} \mathrm{II} / \mathrm{H}]=-1.06$, and $v_{t}=1.3 \mathrm{~km} \mathrm{~s}^{-1}$ (Table 5). The gravity value is, however, too low for its location in the CMD, suggesting veiling effects. In fact, a probable hypothesis for B117 is that it is a binary system, composed of a giant plus a BHB star. In this case, in order to obtain its color shift, the BHB star would have $I \sim 15.5$ and $V-I \sim 0.7$. Such a companion would contaminate the spectrum of the RGB star significantly, due to a rather small 1 mag difference, so that veiling would affect the spectrum, and maybe cause a line contamination as well if the two stars have the same radial velocity.

For these reasons we do not report its abundances, except for one very clear feature, which is a very strong barium line that gives a high barium abundance of $[\mathrm{Ba} / \mathrm{Fe}] \approx+0.7$. Star $\mathrm{B} 117$ could be a barium star, i.e., a binary star showing enhancements of $s$-elements and carbon. For this star, the CN lines are too shallow and cannot be measured, whereas the La II $\lambda 6390.48$ region has a low $\mathrm{S} / \mathrm{N}$; the line is too shallow and does not allow us to decide between ratios of $[\mathrm{La} / \mathrm{Fe}]=-0.2$ to +0.2 , but it is not incompatible with an enhancement.

\subsection{Errors}

Final abundances reported in Table 9 were obtained by averaging the abundances resulting from measured lines. The cluster abundances are a mean of the five stars for each element, and the error given is the internal error, corresponding to the dispersion around the mean. 
TABLE 6

Atomic Lines Used and Abundance Ratios Relative to Fe

\begin{tabular}{|c|c|c|c|c|c|c|c|c|c|}
\hline \multirow[b]{2}{*}{ Species } & \multirow{2}{*}{$\begin{array}{c}\lambda \\
(\AA)\end{array}$} & \multirow{2}{*}{$\begin{array}{c}\chi_{\mathrm{ex}} \\
(\mathrm{eV})\end{array}$} & \multirow[b]{2}{*}{$C_{6}$} & \multirow[b]{2}{*}{$\log g f$} & \multicolumn{5}{|c|}{$[\mathrm{X} / \mathrm{Fe}]$} \\
\hline & & & & & B11 & B64 & B73 & F42 & F97 \\
\hline$[\mathrm{O}$ I $] \ldots .$. & 6363.79 & 0.00 & $\ldots$ & -10.25 & +0.3 : & +0.3 : & +0.2 : & $\ldots$ & +0.70 \\
\hline  & 6154.230 & 2.10 & $0.90 \mathrm{E}-31$ & $-1.56^{\mathrm{a}}$ & 0.00 & 0.00 & -0.20 & 0.00 & $\ldots$ \\
\hline 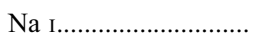 & 6160.753 & 2.10 & $0.30 \mathrm{E}-31$ & $-1.26^{\mathrm{a}}$ & -0.20 & -0.15 & 0.00 & 0.00 & -0.2 : \\
\hline 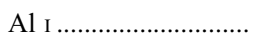 & 6696.020 & 3.14 & $0.30 \mathrm{E}-31$ & $-1.48^{b}$ & 0.00 & +0.15 & -0.10 & 0.00 & 0.00 \\
\hline 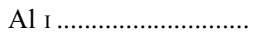 & 6698.670 & 3.14 & $0.30 \mathrm{E}-31$ & $-1.78^{b}$ & 0.00 & $\ldots$ & -0.10 & +0.10 & $\ldots$ \\
\hline 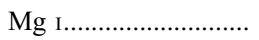 & 6318.720 & 5.11 & $0.30 \mathrm{E}-30$ & $-2.10^{\mathrm{c}}$ & +0.20 & +0.20 & +0.20 & +0.30 & +0.15 \\
\hline 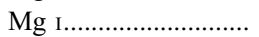 & 6319.242 & 5.11 & $0.30 \mathrm{E}-31$ & $-2.36^{\mathrm{c}}$ & +0.20 & +0.20 & $+0.3:$ & +0.30 & +0.30 \\
\hline Mg I........................ & 6319.490 & 5.11 & $0.30 \mathrm{E}-31$ & $-2.90^{\mathrm{d}}$ & +0.20 & +0.20 & +0.3 : & +0.30 & $\ldots$ \\
\hline Mg I........................ & 6765.450 & 5.75 & $0.30 \mathrm{E}-31$ & $-1.94^{\mathrm{b}}$ & $\ldots$ & +0.20 : & $\ldots$ & $\ldots$ & $\ldots$ \\
\hline  & 6142.494 & 5.62 & $0.30 \mathrm{E}-31$ & $-1.58^{\mathrm{d}}$ & +0.35 & +0.30 & +0.30 & +0.2 : & +0.25 \\
\hline 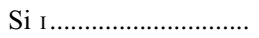 & 6145.020 & 5.61 & $0.30 \mathrm{E}-31$ & $-1.50^{\mathrm{d}}$ & +0.35 & +0.30 & +0.30 & 0.0 : & +0.25 \\
\hline 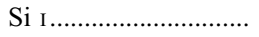 & 6155.142 & 5.62 & $0.30 \mathrm{E}-30$ & $-0.85^{\mathrm{c}}$ & +0.30 & +0.15 & +0.20 & +0.10 & $0.0 ?$ \\
\hline 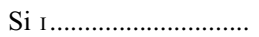 & 6237.328 & 5.61 & $0.30 \mathrm{E}-30$ & $-1.10^{\mathrm{d}}$ & +0.10 & +0.20 & +0.30 & $+0.2:$ & +0.25 \\
\hline 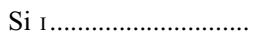 & 6243.823 & 5.61 & $0.30 \mathrm{E}-32^{\mathrm{e}}$ & $-1.30^{\mathrm{f}}$ & +0.10 & +0.30 & +0.20 & +0.2 : & +0.25 \\
\hline 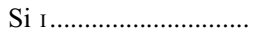 & 6414.987 & 5.87 & $0.30 \mathrm{E}-30$ & $-1.13^{\mathrm{c}}$ & +0.30 & +0.30 & +0.30 & $\ldots$ & $+0.35 ?$ \\
\hline 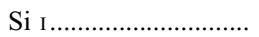 & 6721.844 & 5.86 & $0.90 \mathrm{E}-30$ & $-1.17^{\mathrm{b}}$ & +0.10 & $\ldots$ & +0.30 & +0.3 : & $\ldots$ \\
\hline 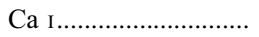 & 6156.030 & 2.52 & $0.30 \mathrm{E}-31$ & $-2.59^{d}$ & +0.30 & 0.0 : & $\ldots$ & -0.05 & +0.15 \\
\hline 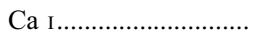 & 6161.295 & 2.51 & $5.98 \mathrm{E}-31^{\mathrm{e}}$ & $-1.42^{\mathrm{d}}$ & 0.00 & 0.00 & +0.15 & -0.15 & 0.0 : \\
\hline 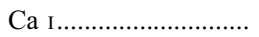 & 6162.167 & 2.52 & $1.95 \mathrm{E}-31^{\mathrm{e}}$ & $-0.09^{\mathrm{a}}$ & 0.00 & 0.00 & +0.15 & -0.15 & 0.0 : \\
\hline 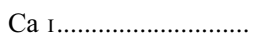 & 6166.440 & 2.52 & $1.0 \mathrm{E}-32^{\mathrm{e}}$ & $-1.1^{\mathrm{d}}$ & 0.00 & 0.00 & 0.00 & -0.15 & +0.15 \\
\hline 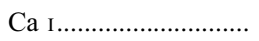 & 6169.044 & 2.52 & $5.95 \mathrm{E}-32^{\mathrm{e}}$ & $-0.70^{\mathrm{c}}$ & 0.00 & 0.00 & +0.15 & 0.00 & 0.0 : \\
\hline 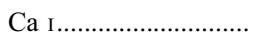 & 6169.564 & 2.52 & $5.95 \mathrm{E}-32^{\mathrm{e}}$ & $-0.55^{\mathrm{d}}$ & 0.00 & 0.00 & +0.15 & 0.00 & 0.0 : \\
\hline 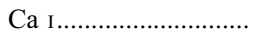 & 6439.080 & 2.52 & $5.12 \mathrm{E}-32^{\mathrm{e}}$ & $+0.3^{\mathrm{f}}$ & 0.00 & 0.00 & 0.00 & 0.00 & +0.15 \\
\hline 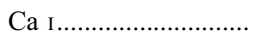 & 6455.605 & 2.52 & $5.06 \mathrm{E}-31^{\mathrm{e}}$ & $-1.35^{\mathrm{a}}$ & +0.15 & +0.15 & +0.10 & -0.05 & +0.15 \\
\hline  & 6464.679 & 2.52 & $5.09 \mathrm{E}-32^{\mathrm{e}}$ & $-2.35^{\mathrm{d}}$ & +0.35 & 0.00 & +0.15 & $\ldots$ & $\ldots$ \\
\hline 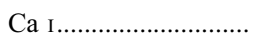 & 6471.668 & 2.52 & $5.09 \mathrm{E}-32^{\mathrm{e}}$ & $-0.59^{\mathrm{a}}$ & +0.35 & +0.15 & 0.00 & 0.00 & +0.15 \\
\hline 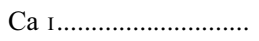 & 6493.788 & 2.52 & $5.05 \mathrm{E}-32^{\mathrm{e}}$ & $-0.20^{\mathrm{g}}$ & 0.00 & +0.10 & +0.15 & 0.00 & 0.0 \\
\hline 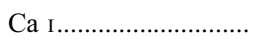 & 6499.654 & 2.52 & $5.05 \mathrm{E}-32^{\mathrm{e}}$ & $-0.82^{h}$ & 0.00 & 0.00 & +0.25 & -0.15 & +0.15 \\
\hline 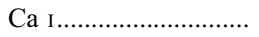 & 6508.846 & 2.52 & $0.30 \mathrm{E}-31$ & $-2.50^{\mathrm{b}}$ & $\ldots$ & 0.00 & $\ldots$ & 0.00 & $\ldots$ \\
\hline Са I.............................. & 6572.779 & 0.00 & $2.62 \mathrm{E}-32^{\mathrm{e}}$ & $-4.29^{\mathrm{a}}$ & 0.00 & 0.00 & +0.10 & +0.15 & +0.15 \\
\hline 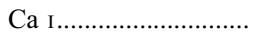 & 6717.687 & 2.71 & $0.70 \mathrm{E}-30$ & $-0.61^{\mathrm{a}}$ & 0.00 & +0.15 & +0.15 & -0.15 & 0.0 \\
\hline Ti I ................................ & 6126.224 & 1.07 & $2.06 \mathrm{E}-32^{\mathrm{e}}$ & $-1.43^{\mathrm{a}}$ & -0.20 & +0.15 & 0.00 & 0.00 & +0.10 \\
\hline Ti ı............................... & 6258.110 & 1.44 & $4.75 \mathrm{E}-32^{\mathrm{e}}$ & $-0.36^{\mathrm{a}}$ & -0.20 & 0.00 & -0.15 & +0.20 & -0.20 \\
\hline Ti I ................................ & 6261.106 & 1.43 & $4.68 \mathrm{E}-32^{\mathrm{e}}$ & $-0.48^{\mathrm{a}}$ & -0.20 & 0.00 & 0.00 & +0.20 & -0.20 \\
\hline 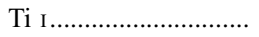 & 6303.767 & 1.44 & $1.53 \mathrm{E}-32^{\mathrm{e}}$ & $-1.57^{\mathrm{a}}$ & $\ldots$ & $\ldots$ & $\ldots$ & +0.20 & $\ldots$ \\
\hline 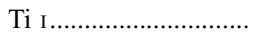 & 6312.238 & 1.46 & $1.51 \mathrm{E}-32^{\mathrm{e}}$ & $-1.69^{d}$ & +0.20 & +0.15 : & 0.0 : & +0.10 & +0.15 \\
\hline 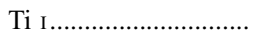 & 6336.113 & 1.44 & $0.30 \mathrm{E}-31$ & $-1.74^{\mathrm{a}}$ & 0.0 : & $\ldots$ & 0.0 : & 0.00 & $0.0:$ \\
\hline 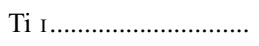 & 6508.154 & 1.43 & $1.46 \mathrm{E}-32^{\mathrm{e}}$ & $-2.05^{\mathrm{b}}$ & $\ldots$ & $\ldots$ & 0.0 & +0.20 & $\ldots$ \\
\hline Ti I ................................ & 6554.238 & 1.44 & $2.72 \mathrm{E}-32^{\mathrm{e}}$ & $-1.15^{\mathrm{d}}$ & 0.00 & +0.15 & 0.00 & +0.10 & 0.0 \\
\hline Ti ı ............................... & 6556.077 & 1.46 & $2.74 \mathrm{E}-32^{\mathrm{e}}$ & $-1.07^{\mathrm{a}}$ & 0.00 & +0.10 & 0.00 & +0.10 & 0.0 \\
\hline 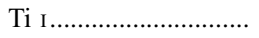 & 6599.113 & 0.90 & $2.94 \mathrm{E}-32^{\mathrm{e}}$ & $-2.09^{\mathrm{a}}$ & +0.20 & 0.00 & +0.05 & +0.20 & 0.00 \\
\hline Ti I ................................ & 6743.127 & 0.90 & $0.30 \mathrm{E}-31$ & $-1.68^{\mathrm{c}}$ & 0.00 & 0.0 & -0.15 & 0.00 & +0.15 \\
\hline 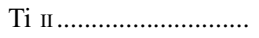 & 6491.580 & 2.06 & $0.30 \mathrm{E}-31$ & $-1.79^{g}$ & +0.10 & +0.20 & +0.20 & +0.20 & +0.2 : \\
\hline 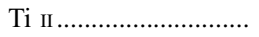 & 6559.576 & 2.05 & $0.30 \mathrm{E}-31$ & $-2.02^{\mathrm{g}}$ & +0.3 : & 0.00 & +0.20 & +0.20 & +0.1 : \\
\hline 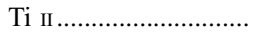 & 6606.970 & 2.06 & $0.30 \mathrm{E}-31$ & $-2.64^{\mathrm{g}}$ & +0.30 & $\ldots$ & +0.20 & +0.20 & $\ldots$ \\
\hline 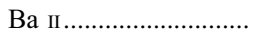 & 6141.727 & 0.70 & $\ldots$ & hfs & 0.00 & 0.00 & +0.20 & 0.00 & +0.20 \\
\hline 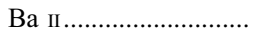 & 6496.908 & 0.60 & $\ldots$ & hfs & +0.15 & +0.20 & +0.20 & +0.20 & +0.20 \\
\hline La II ............................. & 6390.480 & 0.32 & $\ldots$ & hfs & 0.0: & 0.00 & 0.0: & +0.10 & $\ldots$ \\
\hline 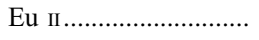 & 6645.127 & 1.38 & $\ldots$ & hfs & +0.40 & +0.40 : & +0.40 & +0.20 & +0.40 \\
\hline
\end{tabular}

NoTE.-The label "hfs" indicates hyperfine structure.

${ }^{a}$ NIST Atomic Spectra Database. See footnote 11.

b McWilliam \& Rich (1994).

c Barbuy et al. (1999).

d Revised in this work.

e Damping constants computed as described in Barbuy et al. (2006b).

${ }^{f}$ Bensby et al. (2003).

g VALD (Kupka et al. 1999).

${ }^{\text {h }}$ Brown \& Wallerstein (1992). 
TABLE 7

Ti il Oscillator Strengths

\begin{tabular}{|c|c|c|c|c|c|c|c|}
\hline $\begin{array}{l}\text { Ti II Line } \\
\text { (1) }\end{array}$ & $\begin{array}{c}\text { VALD } \\
\text { (Kupka et al. 1999) } \\
\text { (2) }\end{array}$ & $\begin{array}{l}\text { NIST ASD } \\
\text { (3) }\end{array}$ & $\begin{array}{c}\text { McWilliam \& Rich } \\
\text { (1994) } \\
\text { (4) }\end{array}$ & $\begin{array}{l}\text { Bensby et al. } \\
\text { (2004) } \\
\text { (5) }\end{array}$ & $\begin{array}{l}\text { Zoccali et al. } \\
\text { (2004) } \\
(6)\end{array}$ & $\begin{array}{c}\text { Barbuy et al. } \\
(2006 \mathrm{~b}) \\
(7)\end{array}$ & $\begin{array}{c}\text { This Paper } \\
\text { (8) }\end{array}$ \\
\hline $6491.580 \ldots \ldots \ldots \ldots$ & -1.79 & -2.10 & $\ldots$ & $\ldots$ & -2.10 & -1.85 & -1.95 \\
\hline $6559.576 \ldots \ldots \ldots \ldots$ & -2.02 & $\ldots$ & -2.48 & $\ldots$ & -2.48 & -2.25 & -2.30 \\
\hline $6606.970 \ldots \ldots \ldots \ldots$ & -2.79 & -2.79 & $\ldots$ & -2.76 & -2.79 & -2.79 & -2.79 \\
\hline
\end{tabular}

TABLE 8

Globular Clusters of Moderate Metallicity with a Blue Horizontal Branch

\begin{tabular}{|c|c|c|c|c|c|c|}
\hline Cluster & $\alpha(\mathbf{J} 2000.0)$ & $\delta(\mathrm{J} 2000.0)$ & $\begin{array}{c}l \\
(\mathrm{deg})\end{array}$ & $\begin{array}{c}b \\
(\operatorname{deg})\end{array}$ & {$[\mathrm{Fe} / \mathrm{H}]$} & Ref. \\
\hline NGC $6558 \ldots \ldots \ldots . . . . . . .$. & 181017.6 & -314550 & 0.20 & -6.02 & -0.9 & 1 \\
\hline HP 1 & 173105.2 & -294458.54 & 357.42 & 2.12 & -1.0 & 2 \\
\hline AL 3 & 181406.6 & -283806 & 3.36 & -5.27 & -1.3 & 3 \\
\hline 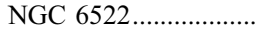 & 180334.08 & $\begin{array}{llll}-30 & 02 & 02.3\end{array}$ & 1.03 & -3.93 & $-1.28 /-1.0$ & 4,5 \\
\hline
\end{tabular}

Noте.-Units of right ascension are hours, minutes, and seconds, and units of declination are degrees, arcminutes, and arcseconds.

ReFerences.- (1) This work; (2) Barbuy et al. 2006b; (3) Ortolani et al. 2006; (4) Terndrup et al. 1998; (5) Castro et al. 1995. 
TABLE 9

Final Abundance Ratios for Each Star, Mean Values, and Corresponding Internal Errors

\begin{tabular}{|c|c|c|c|c|c|c|c|c|c|c|c|}
\hline Star & {$[\mathrm{Fe} / \mathrm{H}]$} & {$[\mathrm{O} / \mathrm{Fe}]$} & {$[\mathrm{Na} / \mathrm{Fe}]$} & {$[\mathrm{Al} / \mathrm{Fe}]$} & {$[\mathrm{Mg} / \mathrm{Fe}]$} & {$[\mathrm{Si} / \mathrm{Fe}]$} & {$[\mathrm{Ca} / \mathrm{Fe}]$} & {$[\mathrm{Ti} / \mathrm{Fe}]$} & {$[\mathrm{Eu} / \mathrm{Fe}]$} & {$[\mathrm{Ba} / \mathrm{Fe}]$} & {$[\mathrm{La} / \mathrm{Fe}]$} \\
\hline $11 \ldots \ldots \ldots \ldots \ldots$ & $-0.98 \pm 0.11$ & +0.3 & -0.10 & 0.00 & +0.20 & +0.23 & +0.08 & +0.04 & 0.4 & +0.07 & 0.0 \\
\hline 64........................ & $-0.98 \pm 0.12$ & +0.3 & -0.07 & +0.15 & +0.20 & +0.26 & +0.04 & +0.08 & 0.4 & +0.10 & 0.0 \\
\hline $73 \ldots \ldots \ldots \ldots \ldots$ & $-0.88 \pm 0.13$ & +0.2 & -0.10 & -0.10 & +0.27 & +0.27 & +0.12 & +0.03 & 0.4 & +0.20 & 0.0 \\
\hline $42 \ldots \ldots \ldots \ldots \ldots \ldots$ & $-1.03 \pm 0.14$ & $\ldots$ & 0.0 & +0.05 & +0.30 & +0.16 & -0.05 & +0.13 & 0.2 & +0.10 & 0.0 \\
\hline 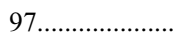 & $-0.96 \pm 0.20$ & +0.7 & -0.20 & 0.0 & +0.23 & +0.23 & +0.08 & 0.00 & 0.4 & +0.20 & $\ldots$ \\
\hline Mean ............ & $-0.97 \pm 0.15$ & $+0.38 \pm 0.17$ & $-0.09 \pm 0.05$ & $+0.02 \pm 0.06$ & $+0.24 \pm 0.05$ & $+0.23 \pm 0.03$ & $+0.05 \pm 0.05$ & $+0.06 \pm 0.04$ & $+0.36 \pm 0.06$ & $+0.13 \pm 0.05$ & 0.0 \\
\hline
\end{tabular}




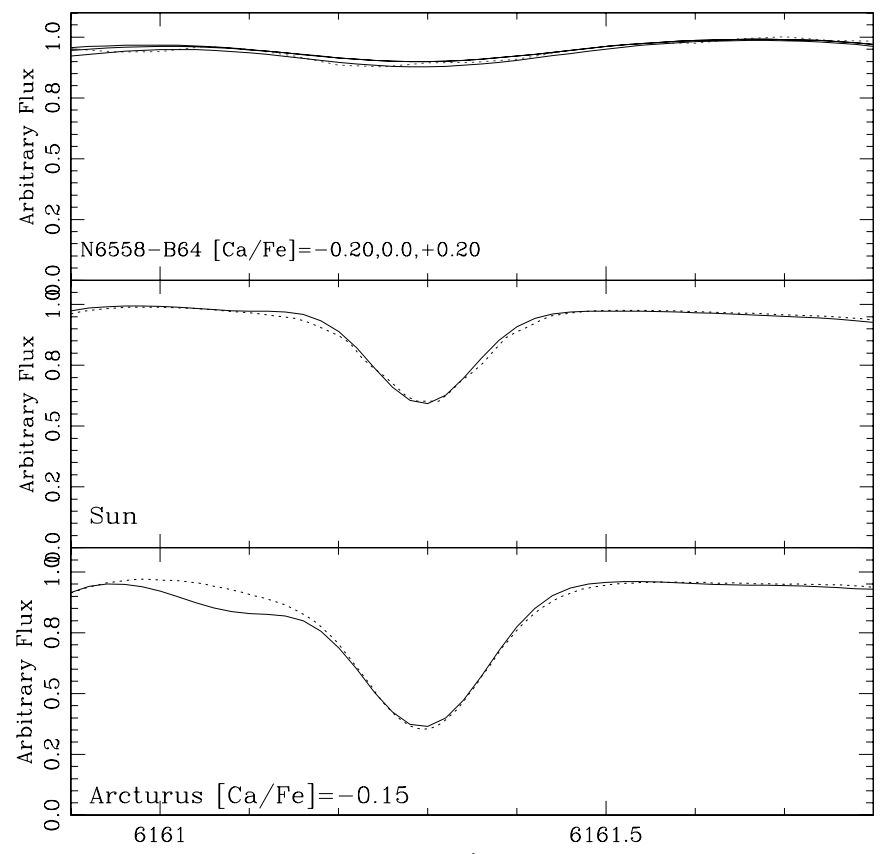

$\lambda(\AA)$

FIG. 4.-Ca I $\lambda 6161.295$ line in the solar, Arcturus, and NGC 6558-B64 star spectra. [See the electronic edition of the Journal for a color version of this figure.]

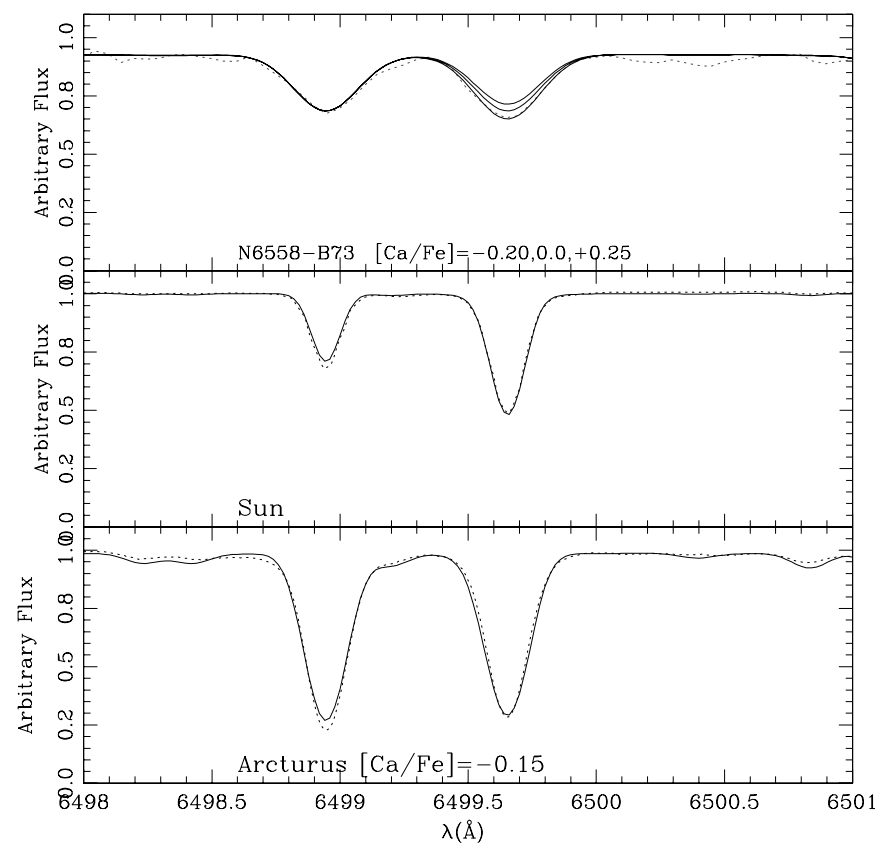

FIG. 5.- Ca I 26499.654 line in the solar, Arcturus, and NGC 6558-B73 star spectra. [See the electronic edition of the Journal for a color version of this figure.]

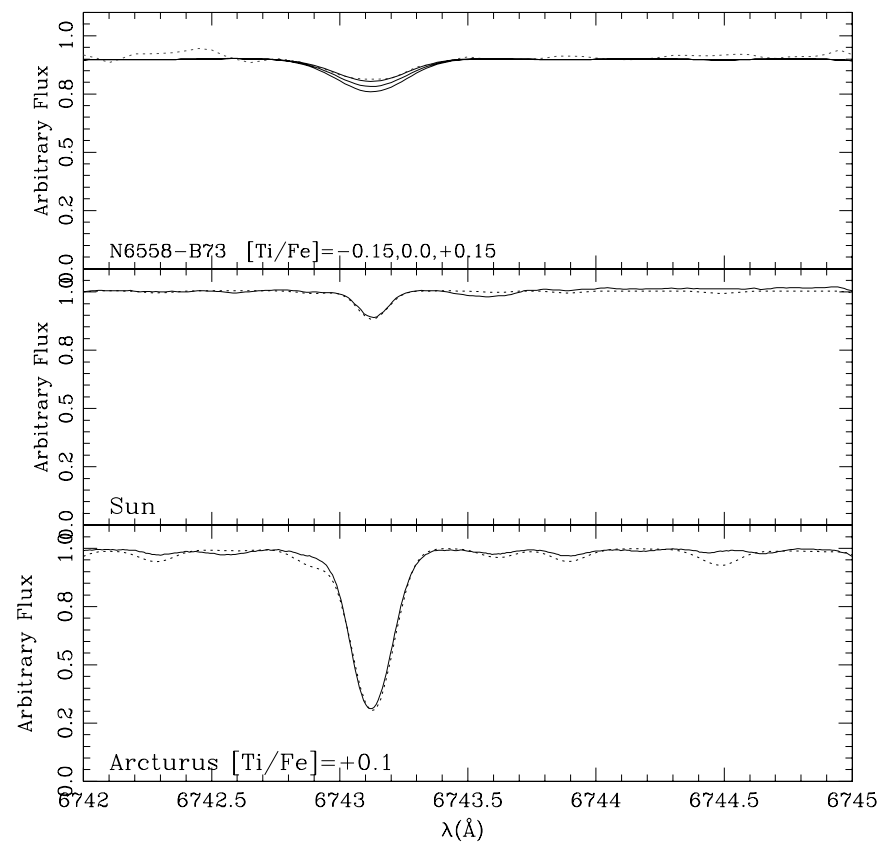

FIG. 6.-Ti I 26743.127 line in the solar, Arcturus, and NGC 6558-B73 star spectra. [See the electronic edition of the Journal for a color version of this figure.]

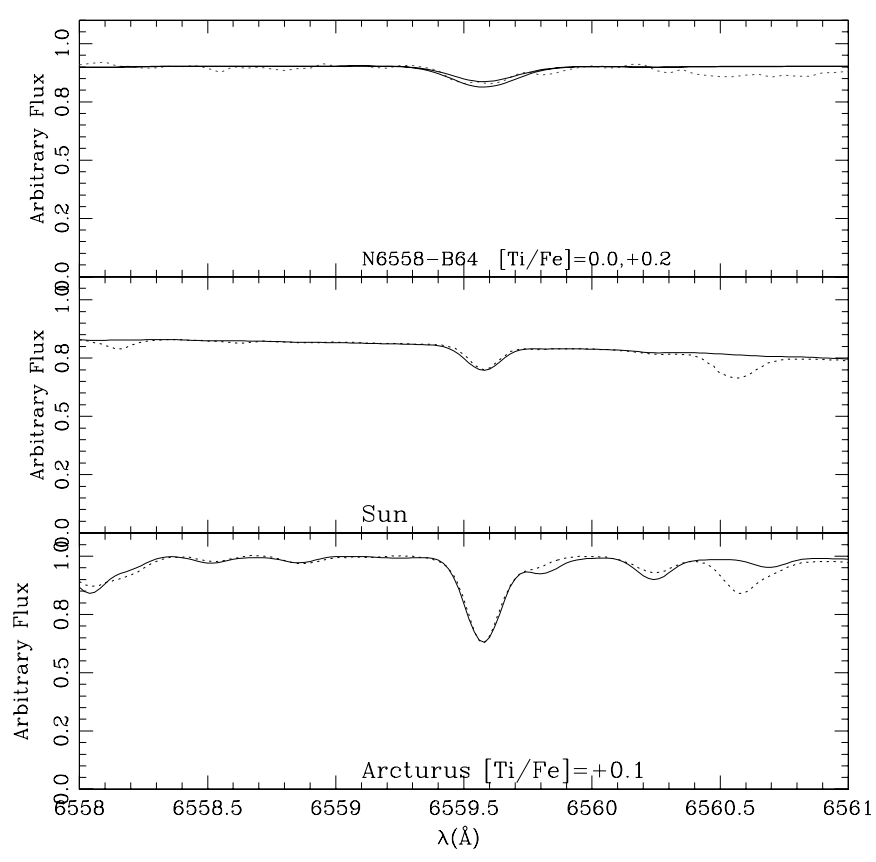

Fig. 7.- - Ti II $\lambda 6559.576$ line in the solar, Arcturus, and NGC 6558-B64 star spectra. [See the electronic edition of the Journal for a color version of this figure.] 
TABLE 10

Abundance Uncertainties and Total Error for Star B11

\begin{tabular}{|c|c|c|c|c|}
\hline $\begin{array}{l}\text { Species } \\
\text { (1) }\end{array}$ & $\begin{array}{l}\Delta T \\
(2)\end{array}$ & $\begin{array}{c}\Delta \log g \\
\text { (3) }\end{array}$ & $\begin{array}{l}\Delta v_{t} \\
(4)\end{array}$ & $\begin{array}{c}\left(\sum x^{2}\right)^{1 / 2} \\
(5)\end{array}$ \\
\hline$[\mathrm{Fe} / \mathrm{H}](\mathrm{I}) \ldots \ldots \ldots \ldots$ & +0.06 & +0.03 & -0.07 & 0.10 \\
\hline$[\mathrm{Fe} / \mathrm{H}](\mathrm{II}) \ldots \ldots \ldots$ & -0.12 & +0.08 & -0.04 & 0.15 \\
\hline$[\mathrm{O} / \mathrm{Fe}] \ldots \ldots \ldots \ldots \ldots$ & 0.00 & +0.05 & 0.00 & 0.05 \\
\hline 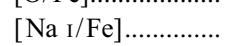 & +0.12 & +0.02 & 0.00 & 0.12 \\
\hline$[\mathrm{Mg}$ I/Fe $] \ldots \ldots \ldots \ldots$ & 0.00 & 0.00 & 0.00 & 0.00 \\
\hline$[\mathrm{Al} \mathrm{I} / \mathrm{Fe}] \ldots \ldots \ldots \ldots$ & +0.08 & 0.00 & +0.01 & 0.08 \\
\hline$[\mathrm{Si}$ I $/ \mathrm{Fe}] \ldots \ldots \ldots \ldots . . . . . . .$. & -0.07 & +0.06 & -0.01 & 0.09 \\
\hline$[\mathrm{Ca}$ I/Fe $] \ldots \ldots \ldots \ldots$ & +0.01 & +0.01 & -0.12 & 0.12 \\
\hline$[\mathrm{Ti}$ I $/ \mathrm{Fe}] \ldots \ldots \ldots \ldots . . . . .$. & +0.05 & +0.03 & -0.04 & 0.06 \\
\hline$[\mathrm{Ti}$ II $/ \mathrm{Fe}] \ldots \ldots \ldots \ldots$ & -0.02 & +0.05 & -0.01 & 0.05 \\
\hline$[\mathrm{Ba}$ II $/ \mathrm{Fe}] \ldots \ldots \ldots \ldots$ & +0.01 & +0.05 & -0.20 & 0.21 \\
\hline$[\mathrm{La}$ II $/ \mathrm{Fe}] \ldots \ldots \ldots \ldots$ & 0.00 & +0.05 & 0.00 & 0.05 \\
\hline$[\mathrm{Eu}$ II $/ \mathrm{Fe}] \ldots \ldots \ldots \ldots$ & -0.02 & +0.06 & 0.00 & 0.06 \\
\hline
\end{tabular}

Note.-These values are for $\Delta T_{\text {eff }}=100 \mathrm{~K}, \Delta \log g=+0.2$, and $\Delta v_{t}=$ $0.3 \mathrm{~km} \mathrm{~s}^{-1}$.

Table 10 gives the uncertainties in abundance ratios, computed for star B11, by allowing a variation of $\Delta T_{\text {eff }}=+100 \mathrm{~K}$, $\Delta \log g=+0.2$, and $\Delta v_{t}=0.3 \mathrm{~km} \mathrm{~s}^{-1}$, which are typical errors in our analysis. The errors are defined as the difference between a parameter derived with the altered model and the nominal model. The total error is given in column (5) as the quadratic sum of all uncertainties.

\section{DISCUSSION}

The metal-poor GCs in the central regions might be the first objects to have formed in the Galaxy. For this reason, highresolution spectroscopy combined with accurate multiband photometry for metal-poor inner GCs provides valuable information on the Galaxy formation scenario.

Among the metal-poor clusters of the inner bulge, only Terzan 4 and HP 1 have high-resolution spectroscopic analyses of individual stars. It is important to collect data for other clusters, and see if the abundance patterns match, in order to understand whether or not they might share a common origin.

High-resolution, near-infrared spectroscopy of four individual stars in Terzan 4 (Origlia \& Rich 2004) revealed an iron content $[\mathrm{Fe} / \mathrm{H}]=-1.6$ and a significant $\alpha$-element enhancement. A rederivation of this cluster distance by Ortolani et al. (2007) placed it at $8 \mathrm{kpc}$ from the Sun, very close to the Galactic center. Likewise, a high-resolution optical spectroscopy analysis of two stars in $\mathrm{HP} 1$ showed $[\mathrm{Fe} / \mathrm{H}]=-1.00 \pm 0.2$ and $[\mathrm{O} / \mathrm{Fe}] \sim[\mathrm{Si} / \mathrm{Fe}] \sim$ +0.3 , while $[\mathrm{Ca} / \mathrm{Fe}]$ and $[\mathrm{Ti} / \mathrm{Fe}]$ are solar (Barbuy et al. 2006b).

The radial velocity of NGC 6558 of $v_{r}=-197 \mathrm{~km} \mathrm{~s}^{-1}$ is high for a bulge cluster. The cluster is projected at $l=0.2^{\circ}$, meaning that we do not expect a high radial velocity for stars rotating with the bulge field stars. The kinematical properties of NGC 6558 seem more compatible with a halo than a bulge population. On the other hand there are exceptions, among which are the probably bulge metal-rich clusters Djorgovski 1 and NGC 6528, showing $v_{r}=-360$ and $200 \mathrm{~km} \mathrm{~s}^{-1}$, respectively (Harris 1996). Furthermore, BHB clusters with metallicities $[\mathrm{Fe} / \mathrm{H}] \approx-1.0$ are only found in the bulge.

In particular, the abundance pattern we find for NGC 6558 turns out to be very similar to that of HP 1 (Fig. 8), with a very similar iron content, $[\mathrm{Fe} / \mathrm{H}]=-0.97([\mathrm{Fe} / \mathrm{H}]=-1.00$ in HP 1). These two clusters also exhibit an extended BHB, with no trace of a red clump. The enhancement of both the $\alpha$-elements $(\mathrm{O}, \mathrm{Mg}$, and $\mathrm{Si})$

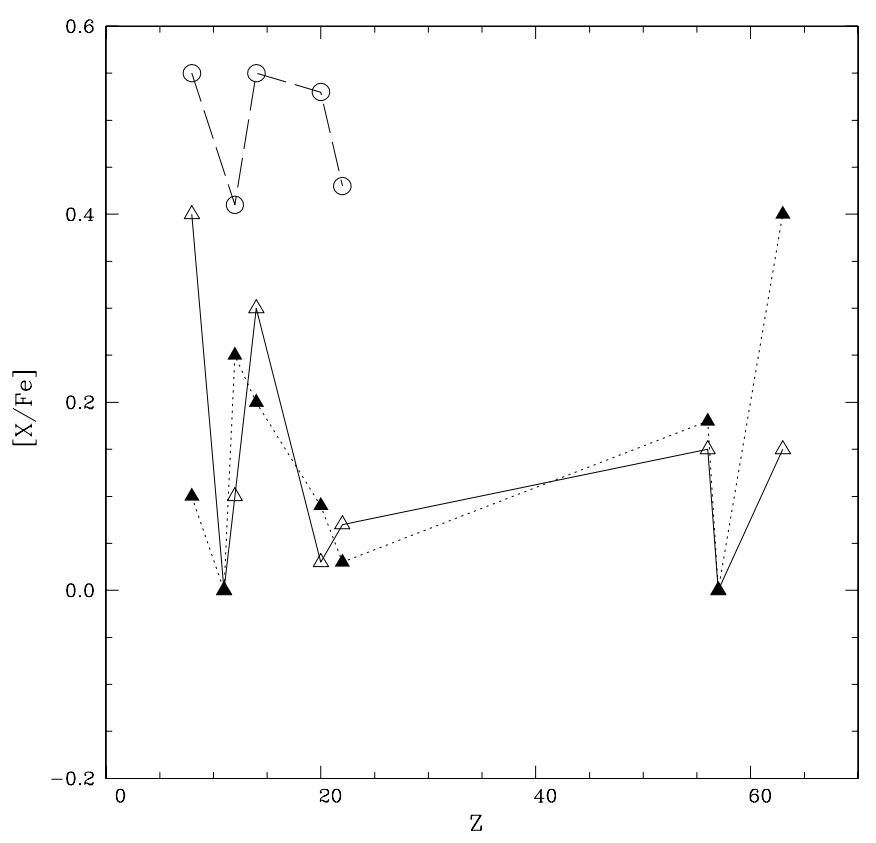

FIG. 8.-Abundance pattern for metal-poor bulge clusters: NGC 6558 ( filled triangles), HP 1 (open triangles), and Terzan 4 (circles).

and the $r$-element $(\mathrm{Eu})$ and the peculiar BHB are consistent with a very early formation, with chemical enrichment dominated by Type II supernovae, and a very old age, as obtained by the isochrone fit shown in Figure 1. Of course, in principle age may not be the only factor producing a BHB in a relatively metal-rich cluster. Recently, it has been shown that several GCs include helium-enriched subpopulations likely to be responsible for the blue extension of their HBs (e.g., Piotto et al. 2007 and references therein). However, at least so far, such helium-enriched populations have been found only among the most massive GCs, with masses in excess of $\sim 10^{6} M_{\odot}$. NGC 6558 is instead a relatively sparse $\mathrm{GC}$, and for this reason we consider age as the most likely factor responsible for its BHB morphology.

The resemblance between HP 1 and NGC 6558 might indicate that they share the same origin. On the other hand, Terzan 4, with its stronger $\alpha$-element enhancement, might be a halo cluster making an excursion into the bulge, given its very low metallicity as compared with the bulge metallicity distribution (Zoccali et al. 2007). Clearly, it would be of great interest to have further analyses of individual stars in other metal-poor bulge and halo GCs in order to understand whether the similarity between NGC 6558 and HP 1 is just a coincidence, or it points to a common origin of all bulge clusters.

B. B. and E. B. acknowledge grants from CNPq and FAPESP. D. M. acknowledges from FONDAP Center for Astrophysics grant 15010003. D. M. also thanks the support from the John Simon Guggenheim Foundation for support. S. O. acknowledges the Italian Ministero dell'Università e della Ricerca Scientifica e Tecnologica (MURST) under the program on Fasi iniziali di Evoluzione dell'Alone e del Bulge Galattico (Italy). This publication makes use of data products from the Two Micron All Sky Survey, which is a joint project of the University of Massachusetts and the Infrared Processing and Analysis Center/California Institute of Technology, funded by the National Aeronautics and Space Administration and the National Science Foundation. 


\section{REFERENCES}

Allende Prieto, C., Lambert, D. L., \& Asplund, M. 2001, ApJ, 556, L63 Alonso, A., Arribas, S., \& Martínez-Roger, C. 1998, A\&AS, 131, 209 1999, A\&AS, 140, 261

Alves-Brito, A., et al. 2006, A\&A, 460, 269

Barbuy, B., Bica, E., \& Ortolani, S. 1998, A\&A, 333, 117

Barbuy, B., Perrin, M.-N., Katz, D., Coelho, P., Cayrel, R., Spite, M., \& Van't Veer-Menneret, C. 2003, A\&A, 404, 661

Barbuy, B., Renzini, A., Ortolani, S., Bica, E., \& Guarnieri, M. D. 1999, A\&A, 341,539

Barbuy, B., et al. 2006a, in Chemical Abundances and Mixing in Stars in the Milky Way and its Satellites, ed. S. Randich \& L. Pasquini (Springer: Berlin), 87

2006b, A\&A, 449, 349

Barklem, P. S., Anstee, S. D., \& O'Mara, B. J. 1998, Publ. Astron. Soc. Australia, 15, 336

Barklem, P. S., Piskunov, N. E., \& O’Mara, B. J. 2000, A\&AS, 142, 467

Bedin, L. R., Piotto, G., Zoccali, M., Stetson, P. B., Saviane, I., Cassisi, S., \& Bono, G. 2000, A\&A, 363, 159

Bekki, K. 2005, ApJ, 626, L93

Bensby, T., Feltzing, S., \& Lundström, I. 2003, A\&A, 410, 527 2004, A\&A, 415, 155

Bessell, M. S. 1979, PASP, 91, 589

Bica, E., Ortolani, S., \& Barbuy, B. 1994, A\&A, 283, 67

Biehl, D. 1976, Ph.D. thesis, Univ. Kiel

Biémont, E., Baudoux, M., Kurucz, R. L., Ansbacher, W., \& Pinnington, A. E. 1991, A\&A, 249, 539

Blanco, V. 1988, AJ, 95, 1400

Brodie, J., \& Strader, J. 2006, ARA\&A, 44, 193

Brown, J. A., \& Wallerstein, G. 1992, AJ, 104, 1818

Carpenter, J. M. 2001, AJ, 121, 2851

Castro, S., Barbuy, B., Bica, E., Ortolani, S., \& Renzini, A. 1995, A\&AS, 111, 17

Côté, P. 1999, AJ, 118, 406

Grevesse, N., \& Sauval, J. 1998, Space Sci. Rev., 85, 161

Gustafsson, B., Edvardsson, B., Eriksson, K., Mizuno-Wiedner, M., Jørgensen, U. G., \& Plez, B. 2003, in ASP Conf. Ser. 288, Stellar Atmosphere Modeling, ed. I. Hubeny, D. Mihalas, \& K. Werner (San Francisco: ASP), 331

Harris, W. E. 1996, AJ, 112, 1487

Idiart, T., Barbuy, B., Perrin, M.-N., Ortolani, S., Bica, E., \& Renzini, A. 2002, A\&A, 381, 472

Kupka, F., Piskunov, N. E., Ryabchikova, T. A., Stempels, H. C., \& Weiss, W. W. 1999, A\&AS, 138, 119

Landolt, A. U. 1992, AJ, 104, 340

Larsen, S. S., Brodie, J. P., Huchra, J. P., Forbes, D. A., \& Grillmair, C. J. 2001, AJ, 121, 2974
Lawler, J. E., Bonvallet, G., \& Sneden, C. 2001a, ApJ, 556, 452

Lawler, J. E., Wickliffe, M. E., den Hartog, E., \& Sneden, C. 2001b, ApJ, 563, 1075

Lecureur, A., Hill, V., Zoccali, M., Barbuy, B., Gómez, A., Minniti, D., Ortolani, S., \& Renzini, A. 2007, A\&A, 465, 799

Lee, J.-W., Carney, B. W., \& Balachandran, S. C. 2004, AJ, 128, 2388

Lee, Y.-W., Demarque, P., \& Zinn, R. 1994, ApJ, 423, 248

McWilliam, A., \& Rich, R. M. 1994, ApJS, 91, 749

Meléndez, J., \& Barbuy, B. 2002, ApJ, 575, 474

Meléndez, J., Barbuy, B., Bica, E., Zoccali, M., Ortolani, S., Renzini, A., \& Hill, V. 2003, A\&A, 411, 417

Minniti, D. 1995, AJ, 109, 1663

Morgan, W. W. 1959, AJ, 64, 432

Origlia, L., \& Rich, R. M. 2004, AJ, 127, 3422

Origlia, L., Rich, R. M., \& Castro, S. 2002, AJ, 123, 1559

Origlia, L., Valenti, E., Rich, R. M., \& Ferraro, F. R. 2005, MNRAS, 363, 897

Ortolani, S., Barbuy, B., Bica, E., Renzini, A., Marconi, G., \& Gilmozzi, R. 1999a, A\&A, 350, 840

Ortolani, S., Barbuy, B., Bica, E., Zoccali, M., \& Renzini, A. 2007, A\&A, 470, 1043

Ortolani, S., Bica, E., \& Barbuy, B. 1993, A\&A, 267, 66

1997, MNRAS, 284, 692

- 1999b, A\&AS, 138, 267 2006, ApJ, 646, L115

Pietrinferni, A., Cassisi, S., Salaris, M., \& Castelli, F. 2004, ApJ, 612, 168 Piotto, G., et al. 2007, ApJ, 661, L53

Rich, R. M., Ortolani, S., Bica, E., \& Barbuy, B. 1998, AJ, 116, 1295

Rich, R. M., et al. 1997, ApJ, 484, L25

Selman, J. F., \& Melnick, J. 2005, A\&A, 443, 851

Skrutskie, M., et al. 2006, AJ, 131, 1163

Sosin, C., et al. 1997, ApJ, 480, L35

Stetson, P. B. 1994, PASP, 106, 250

Strader, J., Brodie, J. P., Cenarro, A. J., Beasley, M. A., \& Forbes, D. A. 2005, AJ, 130, 1315

Terndrup, D. M., Popowski, P., \& Gould, A. 1998, AJ, 115, 1476

Trager, S. C., King, I. R., \& Djorgovski, S. 1995, AJ, 109, 218

van den Bergh, S. 1993, ApJ, 411, 178

Yoon, S.-J., Yi, S. K., \& Lee, Y.-W. 2006, Science, 311, 1129

Zoccali, M., et al. 2004, A\&A, 423, 507

2006, A\&A, 457, L1

2007, in IAU Symp. 241, Stellar Populations as Building Blocks of Galaxies, ed. A. Vazdekis \& R. Peletier (Cambridge: Cambridge Univ. Press), in press 